\title{
Families of Rational and Semirational Solutions of the Partial Reverse Space-Time Nonlocal Mel'nikov Equation
}

\author{
Wei Liu, ${ }^{1}$ Zhenyun Qin $\mathbb{D}^{1},{ }^{2}$ Kwok Wing Chow, ${ }^{3}$ and Senyue Lou ${ }^{4}$ \\ ${ }^{1}$ College of Mathematics and Information Science, Shandong Technology and Business University, Yantai 264005, China \\ ${ }^{2}$ School of Mathematics and Key Laboratory for Nonlinear Mathematical Models and Methods, Fudan University, \\ Shanghai 200433, China \\ ${ }^{3}$ Department of Mechanical Engineering, University of Hong Kong, Pokfulam, Hong Kong \\ ${ }^{4}$ Department of Physics, Ningbo University, Ningbo, Zhejiang, China
}

Correspondence should be addressed to Zhenyun Qin; zyqin@fudan.edu.cn

Received 20 August 2019; Revised 26 February 2020; Accepted 13 March 2020; Published 22 May 2020

Academic Editor: Mohammad Hassan Khooban

Copyright (C) 2020 Wei Liu et al. This is an open access article distributed under the Creative Commons Attribution License, which permits unrestricted use, distribution, and reproduction in any medium, provided the original work is properly cited.

\begin{abstract}
Exact periodic and localized solutions of a nonlocal Mel'nikov equation are derived by the Hirota bilinear method. Many conventional nonlocal operators involve integration over a spatial or temporal domain. However, the present class of nonlocal equations depends on properties at selected far field points which result in a potential satisfying parity time symmetry. The present system of nonlocal partial differential equations consists of two dependent variables in two spatial dimensions and time, where the dependent variables physically represent a wave packet and an auxiliary scalar field. The periodic solutions may take the forms of breathers (pulsating modes) and line solitons. The localized solutions can include propagating lumps and rogue waves. These nonsingular solutions are obtained by appropriate choice of parameters in the Hirota expansion. Doubly periodic solutions are also computed with elliptic and theta functions. In sharp contrast with the local Mel' nikov equation, the auxiliary scalar field in the present set of solutions can attain complex values. Through a coordinate transformation, the governing equation can reduce to the Schrödinger-Boussinesq system.
\end{abstract}

\section{Introduction}

Nonlinear evolution equations (NLEEs) are widely applicable in a variety of intriguing phenomena in physical sciences and engineering, e.g., biophysics, condensed matter, fluids, optics, particle dynamics, and plasma physics [1-8]. Searching for exact solutions of NLEEs is critically important in these applications, as such solutions offer rich knowledge and penetrating insight for the phenomena being modelled by the NLEEs. Many ingenious methods have been devised to solve these NLEEs analytically. Examples include, but are not limited to, the Darboux transformation $[9,10]$, Hirota bilinear method [11], homogeneous balance method [12, 13], inverse scattering transform $[14,15]$, Lie group analysis $[16,17]$, and many other schemes [18-20].

Most of the NLEEs studied in the literature are local equations where the evolution depends only on the local value of the dependent variable and its local space and time derivatives. Nonlocal evolution equations arise in many fields of applications too, where the nonlocal nature frequently comes from a dependence on the global properties, e.g., an integral of the dependent variable [21-23]. Physical scenarios include modulation of nonlinear waves (Whitham's equation) [21] and reaction diffusion systems [22, 23]. Aside from the global operator, the linear portion of the differential equation may be of the forms of the Duffing, heat, or inviscid Burgers equations. Here, we consider another class of nonlocal evolution equations where the intensity of the wave motion depends on values of the dependent variable at points in the far field. Physically, the significance is that this potential displays a parity time symmetry property studied also intensively in quantum mechanics. Recently, Ablowitz and Musslimani [24] have introduced a nonlocal nonlinear Schrödinger (NLS) equation: 


$$
\begin{aligned}
& q_{t}(x, t)-i q_{x x}(x, t) \pm 2 i V(x, t) q(x, t)=0, \\
& V(x, t)=q(x, t) q^{*}(-x, t)
\end{aligned}
$$

which incorporates a parity time (PT) symmetric potential $V$. Fokas extended the nonlocal NLS equation into multidimensional versions and proposed a new integrable nonlocal Davey-Stewartson (DS) equation [25]:

$$
\begin{aligned}
& i A_{t}=A_{x x}+A_{y y}+(\epsilon V-2 Q) A, \\
& Q_{x x}-Q_{y y}=(\epsilon V)_{x x},
\end{aligned}
$$

where

$$
\begin{aligned}
V & =A(x, y, t)[A(-x,-y, t)]^{*}, \quad \varepsilon= \pm 1, \\
\text { or } V & =A(x, y, t)[A(-x, y, t)]^{*}, \quad \varepsilon= \pm 1 .
\end{aligned}
$$

Subsequently, several new nonlocal integrable equations were also investigated [26-43].

Here, the focus is on a set of nonlinear equations which exhibits partial reverse space time in multidimensional setting. Inspired by the works of Ablowitz, Mussliman, and Fokas, we propose a partial reverse space-time nonlocal $\mathrm{Mel}^{\prime}$ nikov equation:

$$
\begin{aligned}
& 3 u_{y y}-u_{x t}-\left[3 u^{2}+u_{x x}+\kappa \phi \phi^{*}(-x, y,-t)\right]_{x x}=0, \\
& i \phi_{y}=u \phi+\phi_{x x},
\end{aligned}
$$

where $u$ and $\phi$ are functions of $x, y$, and $t$. Obviously, by replacing $\phi^{*}(-x, y,-t)$ with $\phi^{*}(x, y, t)$, the nonlocal $\mathrm{Mel}^{\prime}$ nikov equation reduces to the usual $\mathrm{Mel}^{\prime}$ nikov equation:

$$
\begin{aligned}
& 3 u_{y y}-u_{x t}-\left(3 u^{2}+u_{x x}+\kappa|\phi|^{2}\right)_{x x}=0, \\
& i \phi_{y}=u \phi+\phi_{x x} .
\end{aligned}
$$

Here, real $u$ is the long wave component and $\phi$ is the complex valued short wave envelope. As noted by Mel'nikov [44-47], this equation may describe an interaction of long waves with short wave packets, and it could also be considered either as a generalization of the Kadomtsev-Petviashvili (KP) equation with the addition of a complex scalar field or as a generalization of the nonlinear Schrödinger (NLS) equation with a real scalar field [48]. High-order soliton solutions for this equation were calculated by Hase et al. [49]. Rogue wave solutions were derived by $\mathrm{Mu}$ and Qin [50], and general N-dark soliton solutions of the multicomponent $\mathrm{Mel}^{\prime}$ nikov system were investigated by Han et al. [51]. Under the variable transformations,

$$
\begin{aligned}
& \xi=x+t, \\
& \eta=y, \\
& \tau=t, \\
& \kappa=-1,
\end{aligned}
$$

on neglecting the $\tau$-dependence and rewriting $\xi \longrightarrow x, \eta \longrightarrow t$, the nonlocal $\mathrm{Mel}^{\prime}$ nikov equation reduces to the nonlocal Schrödinger-Boussinesq equation:

$$
\begin{aligned}
& 3 u_{t t}-u_{x x}-\left[3 u^{2}+u_{x x}-\phi \phi^{*}(-x, t)\right]_{x x}=0 \\
& i \phi_{t}=u \phi+\phi_{x x} .
\end{aligned}
$$

Rogue wave solutions of the conventional local Schrödinger-Boussinesq equation have been studied by $\mathrm{Mu}$ and Qin [52].

In recent works [37, 38], $(2+1)$-dimensional breather, rational, and semirational solutions of the partially and fully parity-time (PT) symmetric nonlocal DS equations have been reported. Thus, it is natural to seek exact solutions for other partial reverse space-time nonlocal equations too. In this work, we derive families of rational and semirational solutions to the partial reverse spacetime nonlocal Mel'nikov equation (4) by using the Hirota bilinear method.

This paper is organized as follows. In Section 2, soliton and breather solutions are derived by employing Hirota's bilinear method. The unusual feature is that both dependent variables are allowed to be complex valued, leading to novel structures in the auxiliary scalar field. In Section 3, the main theorem on the rational solutions is established, and typical features of these rational solutions are demonstrated. In Section 4, semirational solutions consisting of lumps, breathers, and periodic line waves are generated, and their novel dynamics is also elucidated. In Section 5, doubly periodic solutions are computed in terms of theta and elliptic functions. Our results are summarized in Section 6.

\section{Soliton and Breather Solutions of the Nonlocal Mel'nikov Equation}

To activate the Hirota bilinear method, we formulate the following transformation and allow for nonzero asymptotic condition $(\phi, u) \longrightarrow(1,0)$ as $x, y, t \longrightarrow \infty$ :

$$
\begin{aligned}
& \phi=\frac{g}{f}, \\
& u=2(\log f)_{x x},
\end{aligned}
$$

where $f$ and $g$ are functions with respect to three variables $x, y$, and $t$, which satisfy the condition

$$
f^{*}(-x, y,-t)=f(x, y, t) .
$$

In other words, $f$ can be complex. This is in strong contrast with the local $\mathrm{Mel}^{\prime}$ nikov equation where the auxiliary scalar field must be real. Equation (4) is written in the following bilinear form:

$$
\begin{aligned}
\left(D_{x}^{2}-i D_{y}\right) g \cdot f & =0 \\
\left(D_{x}^{4}+D_{x} D_{t}-3 D_{y}^{2}\right) f \cdot f & =\kappa\left[f^{2}-g g^{*}(-x, y,-t)\right] .
\end{aligned}
$$

Here, the operator $D$ is the Hirota bilinear differential operator [11] defined by 


$$
\begin{aligned}
& P\left(D_{x}, D_{y}, D_{t},\right) F(x, y, t, \ldots) \cdot G(x, y, t, \ldots) \\
& \quad=\left.P\left(\partial_{x}-\partial_{x}, \partial_{y}-\partial_{y}, \partial_{t}-\partial_{t}, \ldots\right) F(x, y, t, \ldots) G\left(x^{\prime}, y^{\prime}, t^{\prime}, \ldots\right)\right|_{x^{\prime}=x, y^{\prime}=y, t^{\prime}=t},
\end{aligned}
$$

where $P$ is a polynomial of $D_{x}, D_{y}, D_{t}, \ldots$.

Following a well-established procedure [11], to obtain $N$ soliton solutions of the nonlocal Mel'nikov equation, we expand $g$ and $f$ as power series of a small parameter $€$ :

$$
\begin{aligned}
& g=1+\varepsilon g_{1}+\varepsilon^{2} g_{2}+\varepsilon^{3} g_{3}+\cdots+\varepsilon^{N} g_{N}, \\
& f=1+\varepsilon f_{1}+\varepsilon^{2} f_{2}+\varepsilon^{3} f_{3}+\cdots+\varepsilon^{N} f_{N},
\end{aligned}
$$

$$
\begin{aligned}
& f=\sum_{\mu=0,1} \exp \left(\sum_{k<j}^{N} \mu_{k} \mu_{j} A_{k j}+\sum_{k=1}^{N} \mu_{k} \eta_{k}\right), \\
& g=\sum_{\mu=0,1} \exp \left(\sum_{k<j}^{N} \mu_{k} \mu_{j} A_{k j}+\sum_{k=1}^{N} \mu_{k}\left(\eta_{k}+i \phi_{k}\right)\right),
\end{aligned}
$$

where

with truncations leading to exact solutions:

$$
\begin{aligned}
\eta_{j} & =i P_{j} x+Q_{j} y+i \Omega_{j} t+\eta_{j}^{0}, \\
\exp \left(i \phi_{j}\right) & =-\frac{P_{j}^{4}-Q_{j}^{2}}{P_{j}^{4}+Q_{j}^{2}}+i \frac{2 P_{j}^{2} Q_{j}}{P_{j}^{4}+Q_{j}^{2}}, \\
\exp \left(A_{j k}\right) & =-\frac{\kappa-\kappa \cos \left(\phi_{j}-\phi_{k}\right)+\left(P_{j}-P_{k}\right)\left(\Omega_{j}-\Omega_{k}\right)-\left(P_{j}-P_{k}\right)^{4}+3\left(Q_{j}-Q_{k}\right)^{2}}{\kappa-\kappa \cos \left(\phi_{j}+\phi_{k}\right)+\left(P_{j}+P_{k}\right)\left(\Omega_{j}+\Omega_{k}\right)-\left(P_{j}+P_{k}\right)^{4}+3\left(Q_{j}+Q_{k}\right)^{2}}
\end{aligned}
$$

and the dispersion relation is

$$
\Omega_{j} P_{j}\left(P_{j}^{4}+Q_{j}^{2}\right)+P_{j}^{4}\left(P_{j}^{4}-2 Q_{j}^{2}-2 \kappa\right)-3 Q_{j}^{4}=0 .
$$

Here, $P_{j}$ and $Q_{j}$ are real parameters and $\eta_{k}^{0}$ is an arbitrary complex constant. The notation $\sum_{\mu=0,1}$ indicates summation over all possible combinations of $\mu_{1}=0,1, \mu_{2}=$ $0,1, \ldots, \mu_{N}=0,1$; the $\sum_{j<k}^{N}$ summation is over all possible combinations of the $N$ elements with the specific condition $j<k$.

Soliton solutions of the nonlocal DS equations may have singularities [37, 38]. Here, by suitable constraints on the parameters $P_{j}, Q_{j}$, and $\eta_{j}^{0}$ in equation (13),

$$
\begin{aligned}
N & =2 n, \\
P_{n+j} & =-P_{j}, \\
Q_{n+j} & =Q_{j}, \\
\eta_{n+j}^{0} & =\eta_{j}^{0^{*}} .
\end{aligned}
$$

General nonsingular $n$-breather solutions can be generated. For instance, with $N=2$ and parameter choices,

$$
\begin{aligned}
& P_{1}=-P_{2}=P, \\
& Q_{1}=Q_{2}=Q, \\
& \eta_{1}^{0}=\eta_{2}^{0}=\eta^{0},
\end{aligned}
$$

where $P, Q$, and $\eta^{0}$ are real, the one breather can be expressed in terms of hyperbolic and trigonometric functions as

$$
\begin{aligned}
\phi & =\frac{g_{0}}{f_{0}}, \\
u & =2\left(\log f_{0}\right)_{x x},
\end{aligned}
$$

where

$$
\begin{aligned}
f_{0}= & \sqrt{M} \cos h \Theta+\cos (P x+\Omega t), \\
g_{0}= & \sqrt{M}\left[\cos ^{2} \psi \cos h \Theta+\sin ^{2} \psi \sin h \Theta\right. \\
& +i \cos \psi \sin \psi(\cos h \Theta-\sin h \Theta)] \\
& +\cos (P x+\Omega t)(\cos \psi+i \sin ) .
\end{aligned}
$$

$M, \Theta, \psi$, and $\eta_{0}$ are defined by

$$
\begin{aligned}
M & =1+\frac{P^{4}}{Q^{2}}, \\
\exp \left(\eta_{0}\right) & =\sqrt{M} \exp \left(\eta^{0}\right), \\
\exp (i \psi) & =\frac{Q^{2}-P^{4}}{P^{4}+Q^{2}}+i \frac{2 P^{2} Q}{P^{4}+Q^{2}}, \\
\Theta & =-\left(Q y+\eta_{0}\right), \\
\Omega & =\frac{P^{4}-2 P^{4} Q^{2}-2 \kappa P^{4}-3 Q^{4}}{P\left(P^{4}+Q^{2}\right)} .
\end{aligned}
$$

The period of $|\phi|$ or $u$ is $2 \pi / P$ along the $x$ direction in the $(x, y)$-plane (Figure 1). In particular, under the parameter constraints, 


$$
\Omega=P,
$$

and the variable transformations defined in equation (6), the one-breather solutions defined in equation (18) reduce to one-breather solutions of the nonlocal Schrödinger-Boussinesq equation. Moreover, the higher-order breather solutions can also be generated from equation (13) under the parameter constraints equation (16), which still maintain the features of being periodic in the $x$ direction and localized in the $y$ direction. For example, taking parameters in equation (13) as

$$
\begin{aligned}
P_{1} & =-P_{2}, \\
P_{3} & =-P_{4}, \\
Q_{1} & =Q_{2}, \\
Q_{3} & =Q_{4}, \\
\eta_{1}^{0} & =\eta_{2}^{* 0}, \\
\eta_{3}^{0} & =\eta_{4}^{* 0},
\end{aligned}
$$

the second-order breather solutions $\phi, u$ can be generated (Figure 2).

In addition to general breather solutions, mixed solutions consisting of breathers and periodic line waves can also be generated by taking parameters in equation (13) as

$$
\begin{aligned}
N & =2 n+1, \\
P_{n+j} & =-P_{j}, \\
Q_{n+j} & =Q_{j}, \\
Q_{2 n+1} & =0, \\
\eta_{n+j}^{0} & =\eta_{j}^{0^{*}} .
\end{aligned}
$$

This family of hybrid solutions is also nonsingular and describes $n$-breather on a background of periodic line waves. The period of the periodic line waves is $2 \pi / P_{2 n+1}$. For example, with $N=3$ and parameter choices in equation (13) as $\left(P_{0}, Q_{0}\right.$, real),

$$
\begin{aligned}
& P_{1}=-P_{2}=P_{0}, \\
& Q_{1}=Q_{2}=Q_{0}, \\
& Q_{3}=0, \\
& \eta_{1}^{0}=\eta_{2}^{0} .
\end{aligned}
$$

A mixed solution consisting of one breather and periodic line waves is obtained (Figure 3 ). The period of the one breather is $2 \pi / P_{0}$, while that of the line waves is $2 \pi / P_{3}$. In this case, $u$ is a complex valued function, which is different from the solutions of the local Mel'nikov equation, where the scalar field must be real. Again, when one takes parameter constraints,

$$
\begin{aligned}
& \Omega_{1}=P_{0}, \\
& \Omega_{2}=-P_{0}, \\
& \Omega_{3}=P_{3},
\end{aligned}
$$

and the variable transformations defined in equation (6), the corresponding mixed solutions reduce to hybrid solutions consisting of one-breather solutions and periodic line waves to the nonlocal Schrödinger-Boussinesq equation.

Another popular method to derive exact solutions to soliton equation theoretically is the Darboux transformation [53-60], but we have demonstrated that the bilinear method is a feasible scheme too. Examples of these solutions include solitons, breathers, rogue waves, and many other types of rational solutions. This alternative is especially valuable as most soliton systems possess bilinear forms.

\section{Rational Solutions of the Nonlocal Mel'nikov Equation}

To generate rational solutions to the nonlocal Mel'nikov equation, a long wave limit is now taken with the provision

$$
\exp \left(\eta_{j}^{0}\right)=-1, \quad 1 \leq j \leq N .
$$

Indeed, under parameter constraints,

$$
\begin{aligned}
& Q_{j}=\lambda_{j} P_{j}, \\
& \eta_{j}^{0}=i \pi, \quad 1 \leq j \leq N,
\end{aligned}
$$

and the limit $P_{j} \longrightarrow 0$ in equation (13), rational solutions can be obtained.

Theorem 1. The partial reverse space-time nonlocal Mel'nikov equation have Nth-order rational solutions:

$$
\phi=\frac{g_{N}}{f_{N}}, \quad u=2\left(\log f_{N}\right)_{x x}
$$

where

$$
\begin{aligned}
f_{N}= & \prod_{j=1}^{N} \theta_{j}+\frac{1}{2} \sum_{j, k}^{N} \alpha_{j k} \prod_{l \neq j, k}^{N} \theta_{l}+\cdots \\
& +\frac{1}{M ! 2^{M}} \sum_{j, k, \ldots, m, n}^{N} \sim \alpha_{j k} \alpha_{s l}^{M} \cdots \alpha_{m n} \prod_{p \neq j, k, \ldots m, n}^{N} \theta_{p}+\cdots, \\
g_{N}= & \prod_{j=1}^{N}\left(\theta_{j}+b_{j}\right)+\frac{1}{2} \sum_{j, k}^{N} \alpha_{j k} \prod_{l \neq j, k}^{N}\left(\theta_{l}+b_{l}\right)+\cdots \\
& +\frac{1}{M ! 2^{M}} \sum_{j, k, \ldots, m, n}^{N} \sim \alpha_{j k} \alpha_{s l}^{M} \cdots \alpha_{m n} \prod_{p \neq j, k, \ldots m, n}^{N}\left(\theta_{p}+b_{p}\right)+\cdots,
\end{aligned}
$$

with 


$$
\begin{aligned}
& \theta_{j}=i x+\lambda_{j} y-i\left(3 \lambda_{j}^{2}+\frac{2 \kappa}{\lambda_{j}^{2}}\right) t, \\
& b_{j}=\frac{2 i}{\lambda_{j}} \\
& a_{j k}=-\frac{4}{\left(\lambda_{j}-\lambda_{k}\right)^{2}},
\end{aligned}
$$

where the two positive integers $j$ and $k$ are not larger than $N$ and $\lambda_{j}$ and $\lambda_{k}$ are arbitrary real constants. As example, the first four terms of equation (29) can be written explicitly as

$$
\begin{aligned}
f_{1}= & \theta_{1}, \\
f_{2}= & \theta_{1} \theta_{2}+a_{12}, \\
f_{3}= & \theta_{1} \theta_{2} \theta_{3}+a_{12} \theta_{3}+a_{13} \theta_{2}+a_{23} \theta_{1}, \\
f_{4}= & \theta_{1} \theta_{2} \theta_{3} \theta_{4}+a_{12} \theta_{3} \theta_{4}+a_{13} \theta_{2} \theta_{4} \\
& +a_{14} \theta_{2} \theta_{3}+a_{23} \theta_{1} \theta_{4}+a_{24} \theta_{1} \theta_{3} \\
& +a_{34} \theta_{1} \theta_{2}+a_{12} a_{34}+a_{13} a_{24}+a_{14} a_{23}, \\
g_{1}= & \theta_{1}+b_{1}, \\
g_{2}= & \left(\theta_{1}+b_{1}\right)\left(\theta_{2}+b_{2}\right)+a_{12}, \\
g_{3}= & \left(\theta_{1}+b_{1}\right)\left(\theta_{2}+b_{2}\right)\left(\theta_{3}+b_{3}\right) a_{12}\left(\theta_{3}+b_{3}\right) \\
& +a_{13}\left(\theta_{2}+b_{2}\right)+a_{23}\left(\theta_{1}+b_{1}\right), \\
g_{4}= & \left(\theta_{1}+b_{1}\right)\left(\theta_{2}+b_{2}\right)\left(\theta_{3}+b_{3}\right)\left(\theta_{4}+b_{4}\right) \\
& +a_{12}\left(\theta_{3}+b_{3}\right)\left(\theta_{4}+b_{4}\right)+a_{13}\left(\theta_{2}+b_{2}\right)\left(\theta_{4}+b_{4}\right) \\
& +a_{14}\left(\theta_{2}+b_{2}\right)\left(\theta_{3}+b_{3}\right)+a_{23}\left(\theta_{1}+b_{1}\right)\left(\theta_{4}+b_{4}\right) \\
& +a_{24}\left(\theta_{1}+b_{1}\right)\left(\theta_{3}+b_{3}\right) \\
& +a_{34}\left(\theta_{1}+b_{1}\right)\left(\theta_{2}+b_{2}\right) \\
& +a_{12} a_{34}+a_{13} a_{24}+a_{14} a_{23} .
\end{aligned}
$$

The abovementioned formulae for $f$ and $g$ will be used to calculate explicit forms of rational solutions.

Remark 1. These rational solutions can be classified into two patterns:

(1) By restricting the parameters,

$$
\begin{aligned}
N & =2 n, \\
\lambda_{n+j} & =-\lambda_{j}, \quad j=1,2,3, \ldots, n,
\end{aligned}
$$

in Theorem 1, the corresponding rational solutions are nonsingular, which are $n$ th-order lumps.

(2) When the parameters do not satisfy constraints defined in equation (32), the corresponding solutions have singularity at the point $(x, y, t)=(0,0,0)$. Thus, hereafter, we just focus on the nonsingular rational solutions.
To demonstrate their typical dynamics, we first consider the first-order lump solutions obtained by taking in equation (29)

$$
\begin{aligned}
N & =2, \\
\lambda_{2} & =-\lambda_{1}, \\
\lambda & =\lambda .
\end{aligned}
$$

In this case, $u$ and $\phi$ can be expressed:

$$
\begin{aligned}
\phi & =1+\frac{g_{2}^{0}}{f_{2}} \\
& =1+\frac{4 i \lambda^{2} y-4}{\left[\lambda x-\left(2 \kappa / \lambda+3 \lambda^{3}\right) t\right]^{2}+\lambda^{4} y^{2}+1}, \\
u & =2\left(\log f_{2}\right)_{x x} \\
& =-4 \frac{\left(\lambda^{2} x-\left(2 k+3 \lambda^{4}\right) t\right)^{2}-\lambda^{6} y^{2}-\lambda^{2}}{\left[\left(\lambda x-\left((2 k / \lambda)+3 \lambda^{3}\right) t\right)^{2}+\lambda^{4} y^{2}+1\right]^{2}} .
\end{aligned}
$$

As discussed in Ref. [38], solutions $\phi$ and $u$ are constant along the $[x(t), y(t)]$ trajectory, where

$$
x-\frac{2 \kappa+\lambda^{4}}{2 \lambda^{2}}, \quad t=0, y=0 .
$$

Moreover, at any fixed time, $(\phi, u) \longrightarrow(1,0)$ when $(x, y)$ becomes unbounded. Hence, these rational solutions are permanent lumps moving on the constant backgrounds.

Hence, we can discuss the patterns of the lump solutions at $t=0$ without loss of generality. In this case, the two solutions $\phi$ and $u$ have critical points:

$$
\begin{aligned}
& A_{1}(x, y)=(0,0), \\
& A_{2}(x, y)=\left(\frac{\sqrt{3}}{\lambda}, 0\right), \\
& A_{3}(x, y)=\left(-\frac{\sqrt{3}}{\lambda}, 0\right),
\end{aligned}
$$

on insisting the first derivatives to vanish $\partial|\phi| / \partial x=\partial|\phi| / \partial y=0(\partial u / \partial x=\partial u / \partial y=0)$. The second derivatives at these three critical points are given by

$$
\begin{array}{r}
\left.|\psi|_{x x}\right|_{A_{1}}=-48 \lambda^{2}, \\
\left.\left(|\psi|_{x x}|\psi|_{y y}-|\psi|_{x y}^{2}\right)\right|_{A_{2}}=\left.\left(|\psi|_{x x}|\psi|_{y y}-|\psi|_{x y}^{2}\right)\right|_{A_{3}}=768 \lambda^{6}, \\
\left.u_{x x}\right|_{A_{1}}=-24 \lambda^{4} \\
\left.\left(u_{x x} u_{y y}-u_{x y}^{2}\right)\right|_{A_{2}}=\left.\left(u_{x x} u_{y y}-u_{x y}^{2}\right)\right|_{A_{3}}=\frac{41}{2} \lambda^{10}
\end{array}
$$

Hence, $A_{1}$ is the maximum point of solutions $\phi$ and $u$ and $A_{2}$ and $A_{3}$ are the minimum points. Thus, there are only bright lumps in the nonlocal $\mathrm{Mel}^{\prime}$ nikov equation, a feature different 


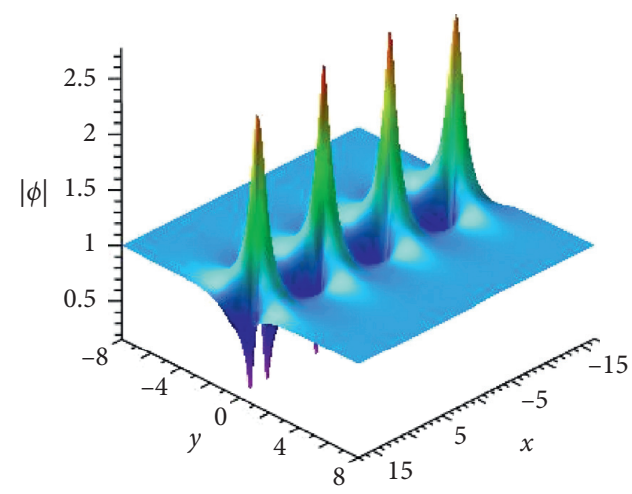

(a)

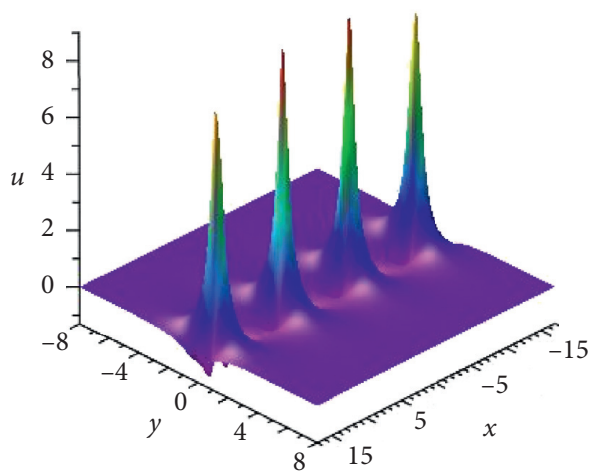

(b)

Figure 1: The one-breather solutions $|\phi|$ and $u$ of the nonlocal Mel'nikov equation given by equation (18) with parameters $\kappa=1 / 2, P=2 / 3, Q=1, \eta^{0}=0$, and $t=0$.

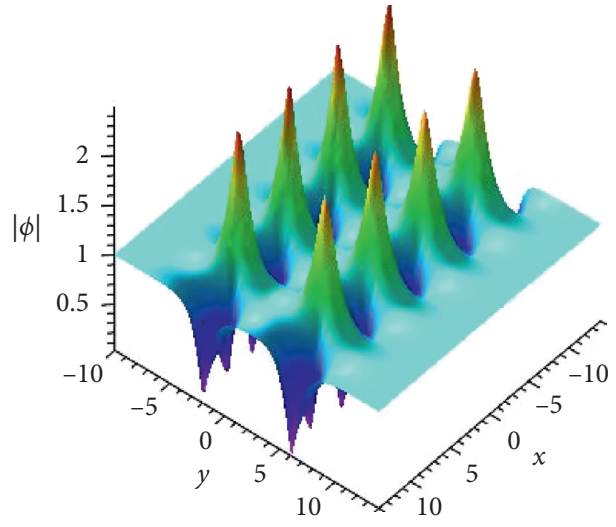

(a)

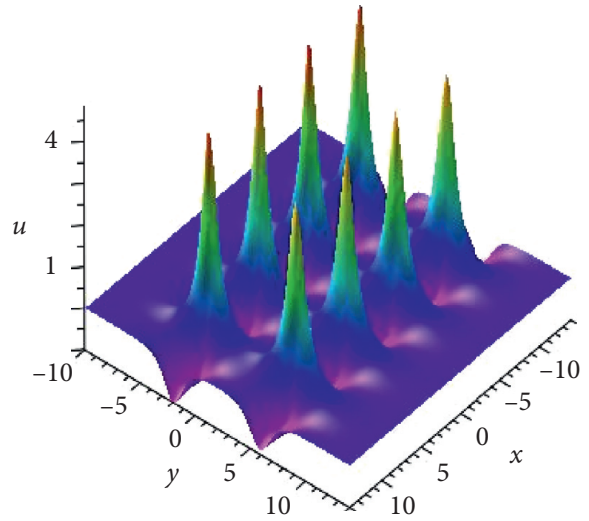

(b)

Figure 2: The two-breather solutions $|\phi|$ and $u$ of the nonlocal $\mathrm{Mel}^{\prime}$ nikov equation with parameters $N=4, P_{1}=1, P_{2}=-1, P_{3}=1$, $P_{4}=-1, \kappa=1 / 2, Q_{2}=2 / 3, Q_{3}=1, Q_{4}=1, \eta_{j}^{0}=0(j=1,2,3,4)$, and $t=0$.

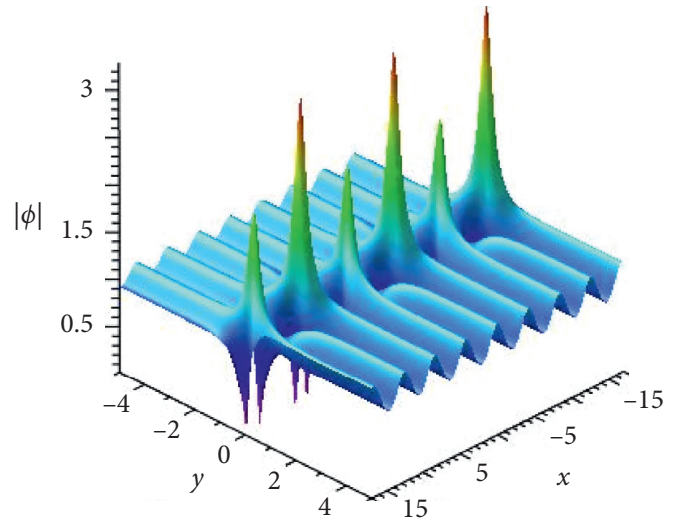

(a)

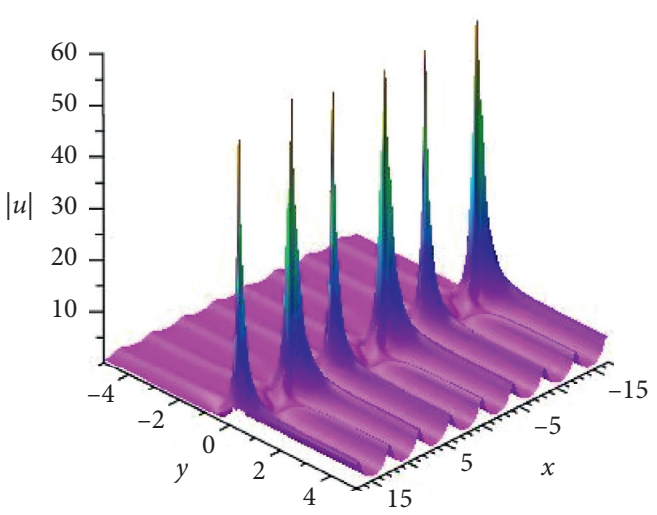

(b)

Figure 3: The mixed solutions $|\phi|$ and $|u|$ consisting of one breather and periodic line waves in the nonlocal Mel'nikov equation given by equation (18) with parameters $\kappa=1, P_{0}=1, Q_{0}=2, P_{3}=1, Q_{3}=0, \eta_{1}^{0}=0, \eta_{2}^{0}=0, \eta_{3}^{0}=-\pi$, and $t=0$.

from the local Mel' nikov equation [45] as the latter possesses three patterns of lumps. Furthermore, by comparison with the rational solutions of the local Mel' nikov equation, the expression of $g_{2}^{0}$ in equation (34) only contains the variable $y$, but the corresponding solutions of the local Mel' nikov equation contain all the variables $x, y$, and $t$ (Figure 4). In particular, on taking 


$$
\begin{aligned}
& \lambda=\frac{\sqrt{6}}{3}, \\
& \kappa=-1,
\end{aligned}
$$

in equations (6) and (34), the two-dimensional lump solutions (34) reduce to the rogue waves of the nonlocal Schrödinger-Boussinesq equation, which can be expressed as

$$
\begin{aligned}
& \psi=1-\frac{24 i t+36}{6 x^{2}+4 t^{2}+9} \\
& u=\frac{24\left(4 t^{2}+9-6 x^{2}\right)}{\left(6 x^{2}+4 t^{2}+9\right)^{2}} .
\end{aligned}
$$

In terms of displacements, $|\psi|$ reaches the maximum amplitude of three (i.e., three times the background amplitude) at the point $(0,0)$, and the minimum amplitude of zero at points $( \pm 3 \sqrt{2} / 2,0) . u$ reaches the maximum amplitude of $8 / 3$ at the point $(0,0)$, and the minimum amplitude of $-(1 / 3)$ at points $( \pm 3 \sqrt{2} / 2,0)$. The function $u$ tends to a zero background in the far field.

Higher-order lumps can be derived for larger $N=2 n(n \geq 2)$ and other parameters which meet the constraints defined in equation (32). The solution then describes the interaction of $n$ fundamental lumps. For example, on taking

$$
\begin{aligned}
& N=4, \\
& \lambda_{1}=-\lambda_{3}, \\
& \lambda_{2}=-\lambda_{4},
\end{aligned}
$$

the second-order lump solutions can be obtained from equation (28), which is

$$
\begin{aligned}
& \phi=\sqrt{2}\left(g_{4} / f_{4}\right), \\
& u=2\left(\log f_{4}\right)_{x x},
\end{aligned}
$$

where $g_{4}$ satisfies equation (31) and

$$
\begin{aligned}
f_{4}= & 36 x^{4}-1260 t x^{3}+\left(15525 t^{2}+36 y^{2}+365\right) x^{2} \\
& +\left(-78750 t^{3}-6590 t-2340 t y^{2}\right) x \\
& +\left(140625 t^{4}+25850 t^{2}+9225 t^{2} y^{2}+144 y^{4}-359 y^{2}+\frac{5329}{9}\right) .
\end{aligned}
$$

Peculiar features occur during the interaction of fundamental lumps (Figure 5). When the two lumps get close to each other, their shapes are deformed. The amplitudes of both entities decrease and remain less than three (the amplitude of the fundamental lump solution). This feature contrasts sharply with that of the nonlocal DSI equation [38], which displays a higher intermediate amplitude during interactions.

For still larger $N$ and parameters satisfying parameters constraints equation (32), higher-order lumps would be obtained, e.g., for

$$
\begin{aligned}
& N=6, \\
& \lambda_{1}=-\lambda_{2}=2, \\
& \lambda_{3}=-\lambda_{4}=\frac{3}{2}, \\
& \lambda_{5}=-\lambda_{6}=1, \\
& \kappa=1,
\end{aligned}
$$

a three-lump solution can be derived:

$$
\begin{aligned}
& \phi=1+\frac{g_{6}^{0}}{f_{6}}, \\
& u=2\left(\log f_{6}\right)_{x x},
\end{aligned}
$$

where $f_{6}$ and $g_{6}^{0}$ are given by equations (A.1) and (A.2) in Appendix A.

These third-order solutions consist of three lumps (Figure 6). The maximum value of $|\phi|$ stays below three at all times.

\section{Semirational Solutions of the Nonlocal Mel'nikov Equation}

To understand resonant behaviours for the nonlocal Mel'nikov equation, we consider several types of semirational solutions. Similar to the derivation of the rational solutions in the last section, semirational solutions are also computed by taking suitable long wave limits. More precisely, a coalescence of wavenumbers at a finite value is taken, instead of the zero wavenumber regime, which would generate purely rational solutions previously. These semirational solutions describe interactions among lumps, breathers, and periodic line waves.

Case 1. A combination of lumps and periodic line waves. The simplest semirational solutions consisting of a lump and periodic line waves are generated from the third-order soliton solutions. Indeed, taking parameters in (13) as

$$
\begin{array}{r}
N=3, \\
Q_{1}=\lambda_{1} P_{1}, \\
Q_{2}=\lambda_{2} P_{2}, \\
\exp \left(\eta_{1}^{0}\right)=\exp \left(\eta_{2}^{0}\right)=-1, \\
Q_{3}=0,
\end{array}
$$

and taking a long wave limit,

$$
P_{1}, P_{2} \longrightarrow 0,
$$

the functions $f$ and $g$ are rewritten as

$$
\begin{aligned}
f= & \left(\theta_{1} \theta_{2}+a_{12}\right)+\left(\theta_{1} \theta_{2}+a_{12}+a_{13} \theta_{2}+a_{23} \theta_{1}+a_{12} a_{23}\right) e^{\eta_{3},} \\
g= & \left(\theta_{1}+b_{1}\right)\left(\theta_{2}+b_{2}\right)+a_{12}+\left[\left(\theta_{1}+b_{1}\right)\left(\theta_{2}+b_{2}\right)+a_{12}\right. \\
& \left.+a_{13}\left(\theta_{2}+b_{2}\right)+a_{23}\left(\theta_{1}+b_{1}\right)+a_{12} a_{23}\right] e^{\eta_{3}+i \phi_{3}},
\end{aligned}
$$




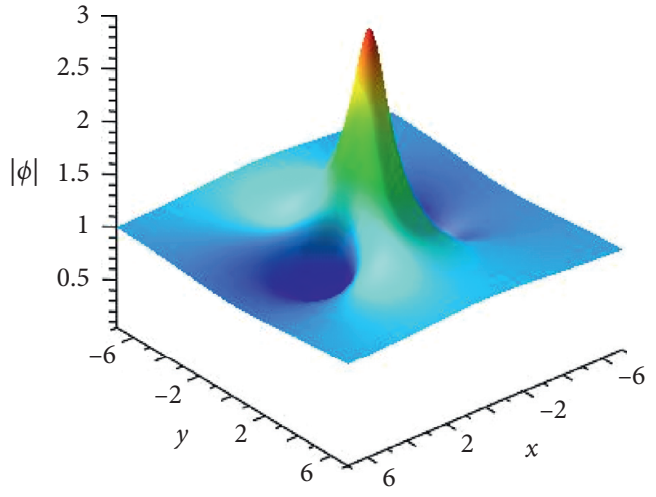

(a)

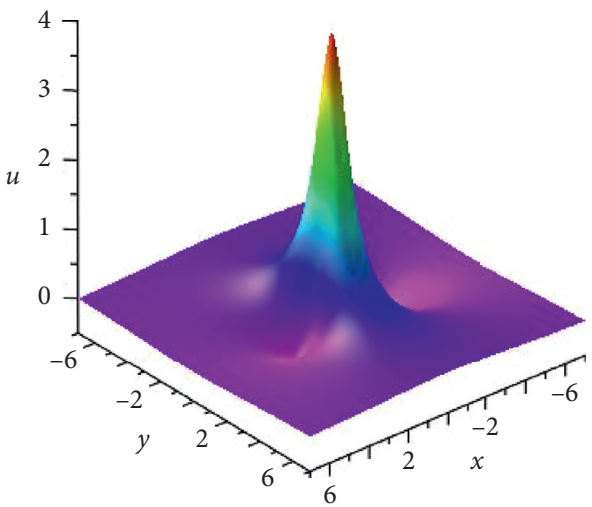

(b)

Figure 4: The one-lump solutions $|\phi|$ and $|u|$ of the nonlocal Mel'nikov equation given by equation (34) with parameters $\kappa=1, \lambda=1$, and $t=0$.

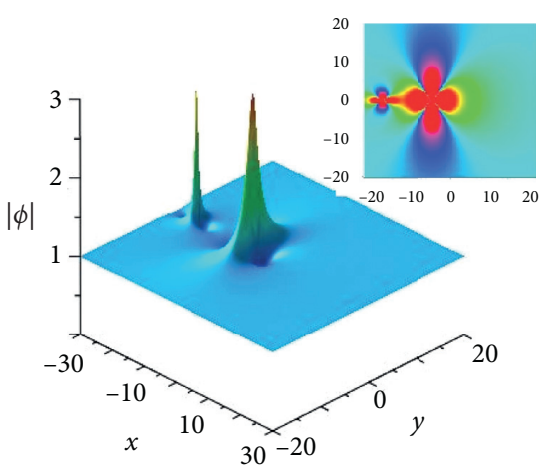

(a)

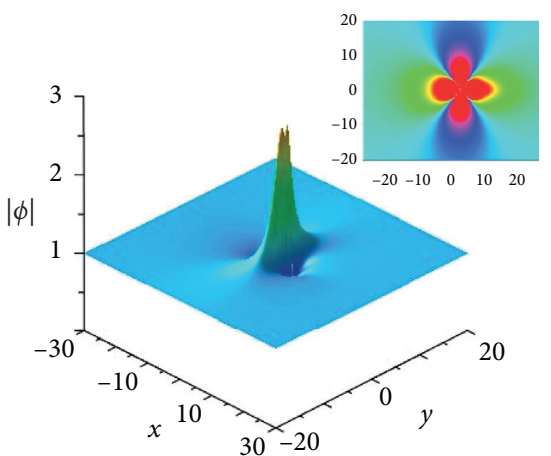

(d)

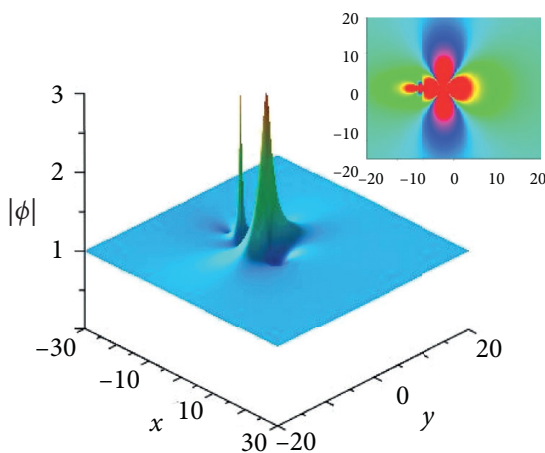

(b)

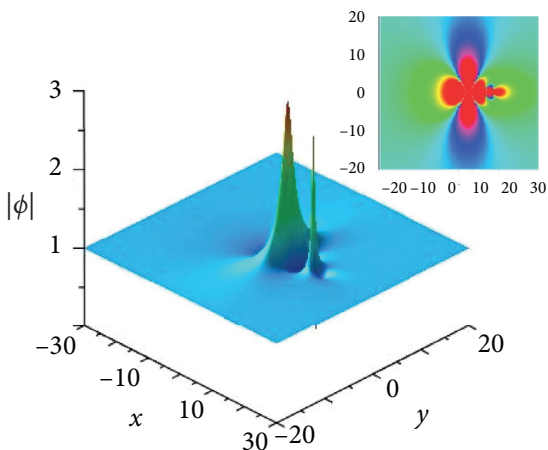

(e)

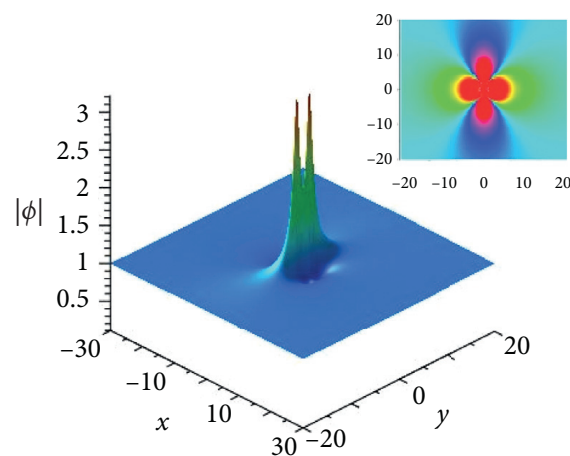

(c)

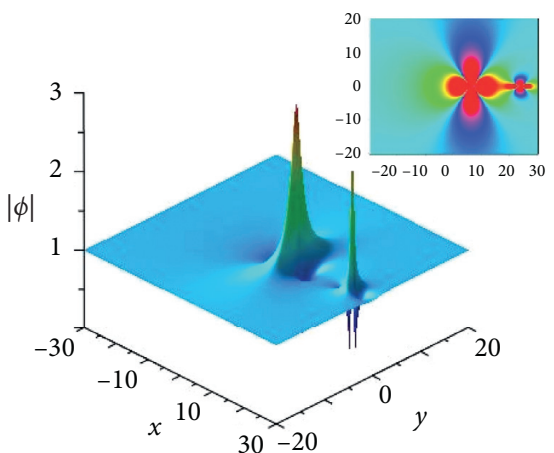

(f)

Figure 5: The interaction of two fundamental lumps at different values in time: (a) $t=-2$. (b) $t=-1$. (c) $t=0$. (d) $t=1 / 2$. (e) $t=1$. (f) $t=2$.

where

$$
a_{s 3}=-\frac{4 P_{3}^{2}}{P_{3}^{2}+\lambda_{s}^{2}}, \quad s=1,2,
$$

and $a_{12}, b_{s}, \phi_{s}, \eta_{3}$ are given by (30) and (14). Furthermore, by taking parameters as

$$
\lambda_{1}=-\lambda_{2}=\lambda_{0}
$$

a semirational solution consisting of a lump and periodic line waves is obtained (Figures 7 and 8 ). The periodic line waves coexisting with a fundamental lump are periodic in $x$ direction and localized in $y$ direction and the period is $2 \pi / P_{3}$. By comparing this type of semirational solutions with that of the nonlocal DSI equation in Ref. [38], the interaction between the lump and periodic line waves can generate either much higher peaks or lower peaks. In Figure 7, the maximum amplitude of the lump can reach 4 (four times the constant background), and thus it is higher than the fundamental lumps. However, in Figure 8, the maximum amplitudes of the lump do not exceed 2 (two times the constant background). In this case, the interaction between the lump and periodic line waves will result in lower peaks. 


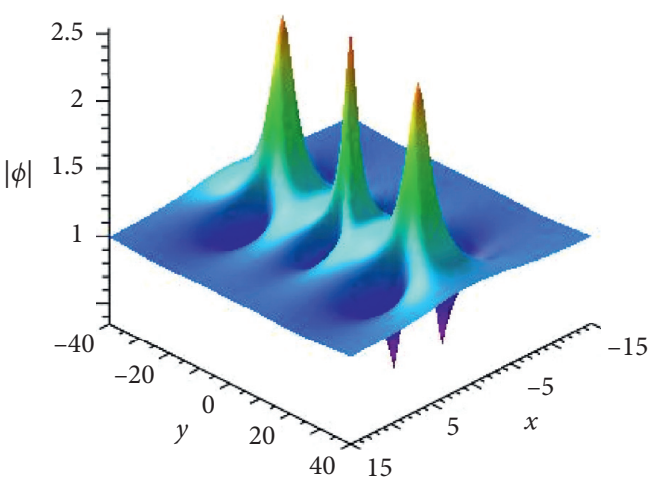

(a)

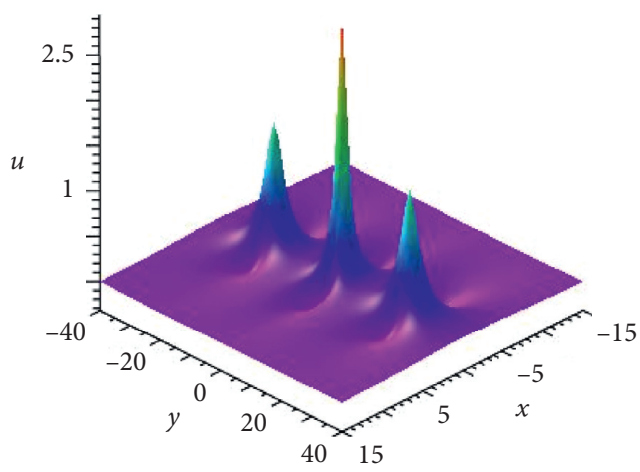

(b)

Figure 6: The third-order lump solutions $|\phi|$ and $u$ defined in equation (44).

In particular, when one takes

$$
\begin{gathered}
\lambda=\frac{\sqrt{6}}{3}, \\
\kappa=-1, \\
\Omega_{3}=P_{3},
\end{gathered}
$$

in equations (6) and (47), we obtain one-dimensional mixed solutions consisting of a fundamental rogue wave and periodic line waves for the nonlocal Schrödinger-Boussinesq equation.

Semirational solutions consisting of more lumps and periodic line waves can also be derived in a similar way for larger $N$. As illustrative examples, we consider a subclass of semirational solutions consisting of two lumps and periodic line waves, which can be derived from 5 -soliton solutions. Indeed, setting the parameters in equation (13) as

$$
\begin{aligned}
N & =5, \\
Q_{j} & =\lambda_{j} P_{j}, \\
\exp \left(\eta_{j}^{0}\right) & =-1, \\
Q_{5} & =0(j=1,2,3,4),
\end{aligned}
$$

and then taking a long wave limit

$$
P_{j} \longrightarrow 0 \quad(j=1,2,3,4)
$$

the functions $f$ and $g$ are changed from exponential functions to a combination of rational and exponential functions, which are explicitly expressed by equation (A.4) in Appendix A. Under the parameter constraints,

$$
\begin{aligned}
& \lambda_{1}=-\lambda_{2}, \\
& \lambda_{3}=-\lambda_{4},
\end{aligned}
$$

a family of semirational solutions describing two lumps on a background of periodic line waves is generated. For typical parameters,

$$
\begin{aligned}
& \lambda_{1}=-\lambda_{2}=1, \\
& \lambda_{3}=-\lambda_{4}=4, \\
& P_{5}=1,
\end{aligned}
$$

$\phi$ and $u$ are shown in Figure 9. The period of these line waves is $2 \pi$. The maximum value of the solution $\phi$ can exceed 4.5 (4.5 times the constant background) at $t=0$, while $|u|$ can reach 12 . In other words, this interaction between these two lumps and periodic line waves can create very high spikes under proper parameter choices.

Case 2. A combination of breathers and periodic line waves

Another type of semirational solutions is a mixed mode consisting of lumps and breathers. Here, we only consider the simplest example, consisting of one lump and one breather. We choose parameters in equation (13) as

$$
\begin{aligned}
N & =4, \\
Q_{1} & =\lambda_{1} P_{1}, \\
Q_{2} & =\lambda_{2} P_{2}, \\
\exp \left(\eta_{1}^{0}\right) & =\exp \left(\eta_{2}^{0}\right)=-1,
\end{aligned}
$$

and take a limit of

$$
P_{1}, P_{2} \longrightarrow 0 \text {. }
$$

Then, functions $f$ and $g$ of solutions to solve the $\mathrm{Mel}^{\prime}$ nikov equation can be rewritten as equation (A.6) in Appendix A. Furthermore, on taking parameters

$$
\begin{aligned}
& \lambda_{1}=-\lambda_{2}, \\
& P_{3}=-P_{4}, \\
& Q_{3}=Q_{4}, \\
& \eta_{3}^{0}=\eta_{4}^{0 *},
\end{aligned}
$$

semirational solutions consisting of a fundamental lump and one breather are obtained (Figure 10). The period of this breather is $2 \pi / P_{3}$. In this case, $u$ is real. For fixed parameters $\eta_{3}^{0}$ and $\eta_{4}^{0}$, the distance between the lump and the breather is not altered during their propagation in the $(x, y)$-plane. In 


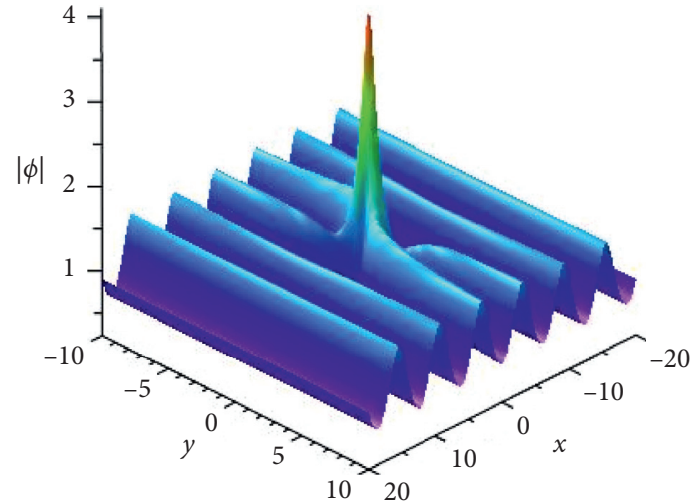

(a)

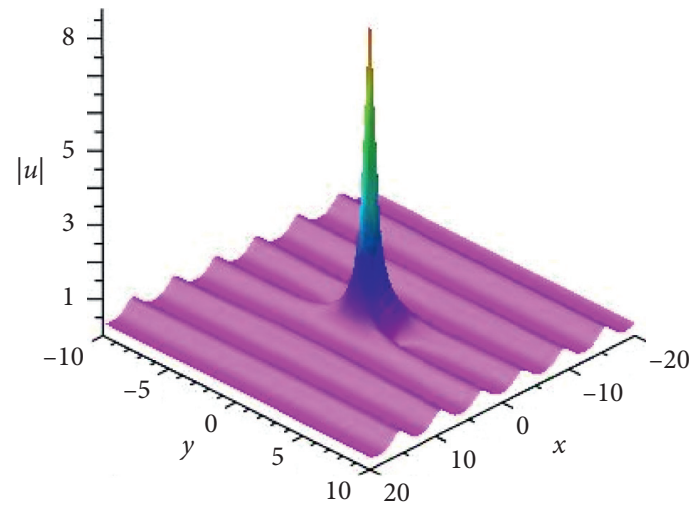

(c)

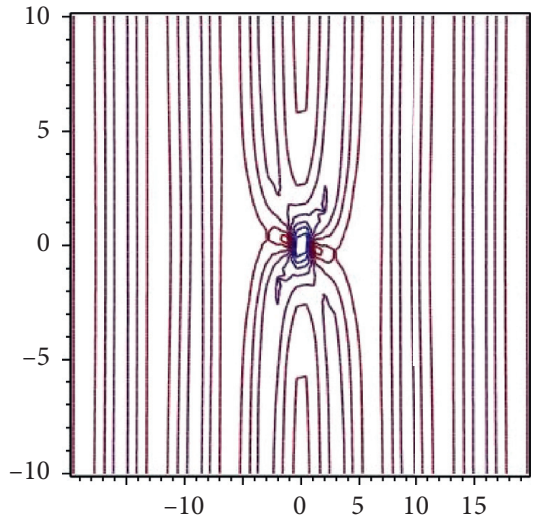

(b)

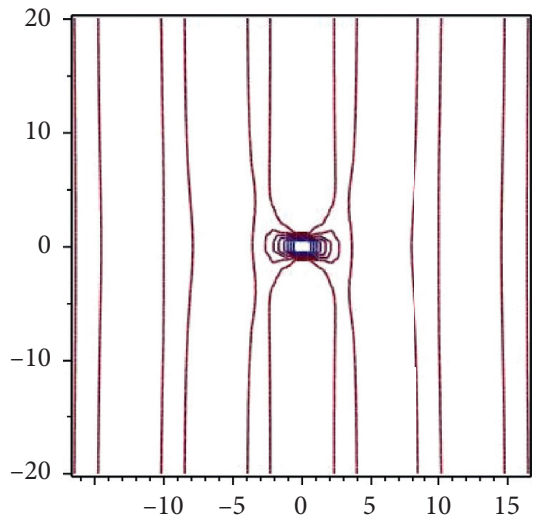

(d)

FiguRe 7: Semirational solutions constituting of a lump and periodic line waves for the local Mel'nikov equation with parameters $\lambda_{0}=1, P_{3}=1$, and $\eta_{3}^{0}=-(\pi / 6)$. The panels (b) and (d) are the contour plots of the panels (a) and (c), respectively.

order to observe the interaction between the lump and breather, we control the location of the breather by adjusting the parameters $\eta_{3}^{0}$, and $\eta_{4}^{0}$. For $\left|\eta_{3}^{0}\right| \longrightarrow 0$, the distance between the lump and the breather tends to zero (Figure 11). The lump immerses itself in the breather (see the panel of $\left.\eta_{3}^{0}=0\right)$. The superposition of the lump and breather excites a peak whose height is less than three times the background amplitude. Moreover, the breather possesses a lower amplitude and a smaller period than the previous example and it propagates stably. Apparently, the wave structure of the lump is destroyed due to the interaction, and energy transfer has occurred between the lump and the breather.

Higher-order semirational solutions consisting of higher-order lumps and higher-order breathers can also be calculated in a similar way with larger $N$ and will be reported in the future.

\section{Doubly Periodic Solutions}

In addition to breather (singly periodic) and rational (algebraically localized) solutions, the evolution system in one spatial dimension, namely, the Schrödinger-Boussinesq equation (equation (7), also admits doubly periodic solutions. The wave profile will be periodic in $x$, but also possesses a distinct period in the $t$ direction as well. In terms of the mathematical techniques employed, theta functions can be expressed as Fourier series with exponentially decreasing coefficients, while elliptic functions can be written as ratios of theta functions. The underlying principle is that the Hirota bilinear derivatives of theta functions can be represented in terms of theta functions themselves $([61,62]$ and Appendix B). Here, we first generalize equation (7) by introducing the auxiliary function $\Phi$ and the real parameter $\sigma$ :

$$
\begin{aligned}
3 u_{t t}-u_{x x}-\left[3 u^{2}+u_{x x}\right]_{x x} & =\sigma\left[\Phi \Phi^{*}(-x, t)\right]_{x x} \\
i \Phi_{t}+\Phi_{x x}+u \Phi & =0
\end{aligned}
$$

with (functions $g$ complex and $f$ real and $\Omega$ being the real angular frequency)

$$
\begin{gathered}
u=2(\log f)_{x x}, \\
\Phi=\frac{\exp (-i \Omega t) g}{f} .
\end{gathered}
$$

The bilinear form for equations (58a) and (58b) can be obtained $\left(C_{0}=a\right.$ constant and $D=$ the Hirota operator): 


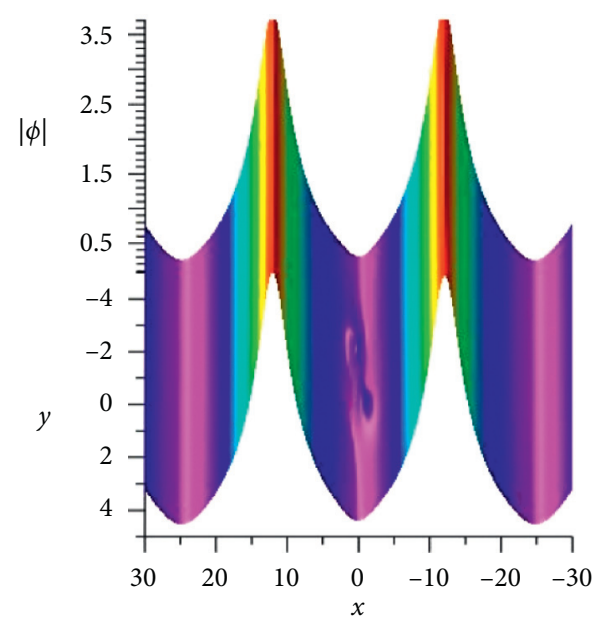

(a)

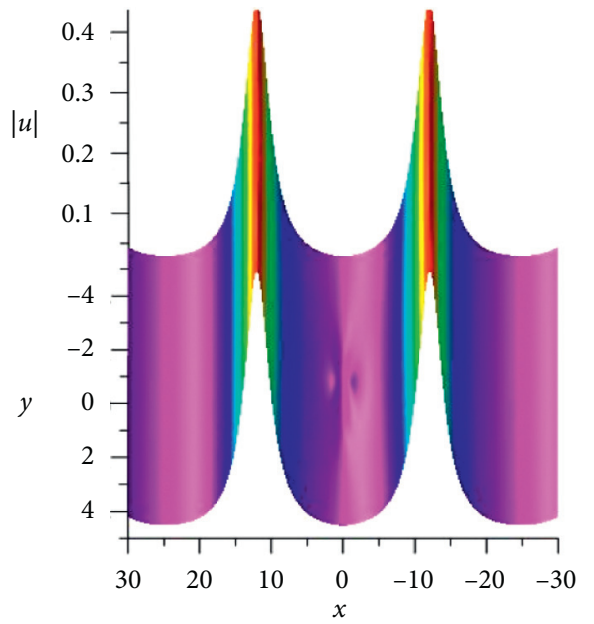

(c)

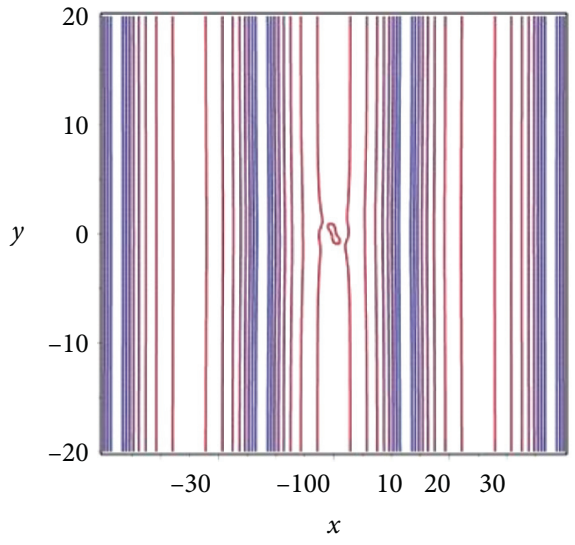

(b)

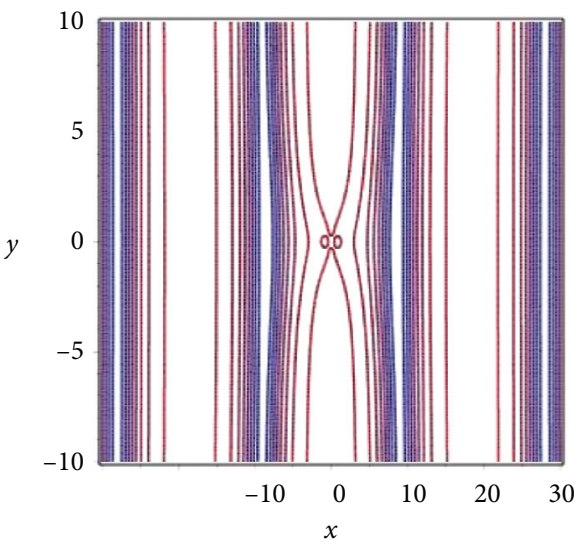

(d)

FIgURE 8: Semirational solutions constituting of a lump and periodic line waves for the nonlocal Mel'nikov equation with parameters $\lambda_{0}=1, P_{3}=1 / 3$, and $\eta_{3}^{0}=-(\pi / 6)$. The panels (b) and (d) are the contour plots of the panels (a) and (c), respectively.

$$
\begin{aligned}
\left(i D_{t}+D_{x}^{2}+\Omega\right) g \cdot f & =0, \\
\left(3 D_{t}^{2}-D_{x}^{2}-D_{x}^{4}-C_{0}\right) f \cdot f & =\sigma g g^{*}(-x, t) .
\end{aligned}
$$

Using combinations of theta functions of $x$ and $t$ with different moduli $\tau$ and $\tau_{1}$ [62], solutions for equations (58a), (58b), and (60) can be expressed as $\left(A_{0}\right.$ and $\Omega$ being real constants to be determined)

$$
\Phi=A_{0}\left[\frac{\theta_{2}\left(\omega t, \tau_{1}\right) \theta_{4}(\alpha x, \tau)+i \theta_{1}\left(\omega t, \tau_{1}\right) \theta_{3}(\alpha x, \tau)}{\theta_{3}\left(\omega t, \tau_{1}\right) \theta_{4}(\alpha x, \tau)+\theta_{4}\left(\omega t, \tau_{1}\right) \theta_{3}(\alpha x, \tau)}\right] \exp (-i \Omega t),
$$

$$
\begin{aligned}
& f=\theta_{3}\left(\omega t, \tau_{1}\right) \theta_{4}(\alpha x, \tau)+\theta_{4}\left(\omega t, \tau_{1}\right) \theta_{3}(\alpha x, \tau), \\
& g=\theta_{2}\left(\omega t, \tau_{1}\right) \theta_{4}(\alpha x, \tau)+i \theta_{1}\left(\omega t, \tau_{1}\right) \theta_{3}(\alpha x, \tau) .
\end{aligned}
$$

From the first bilinear form of equation (60), the period parameter $\omega$ and the angular frequency of the whole wave packet, $\Omega$, are defined in terms of the theta constants as

$$
\begin{aligned}
\omega & =\frac{2 \alpha^{2} \theta_{3}^{2}(0, \tau) \theta_{4}^{2}(0, \tau)}{\theta_{4}^{2}\left(0, \tau_{1}\right)}, \\
\Omega & =-2 \alpha^{2} \frac{\theta_{2}^{\prime \prime}(0, \tau)}{\theta_{2}(0, \tau)} .
\end{aligned}
$$

One constraint relating the two different moduli $\tau$ and $\tau_{1}$ is

$$
\frac{\theta_{3}^{2}\left(0, \tau_{1}\right)}{\theta_{4}^{2}\left(0, \tau_{1}\right)}=\frac{\theta_{3}^{4}(0, \tau)+\theta_{4}^{4}(0, \tau)}{2 \theta_{3}^{2}(0, \tau) \theta_{4}^{2}(0, \tau)}
$$




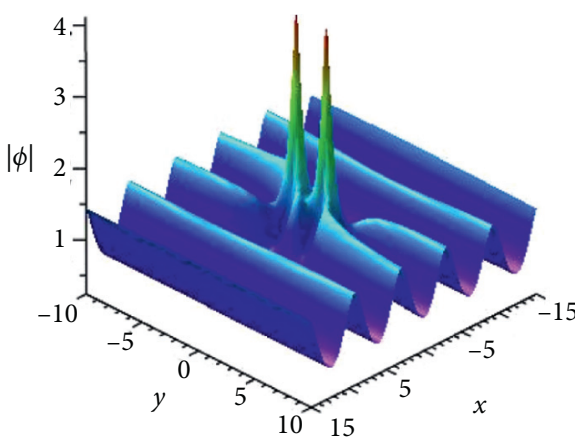

(a)

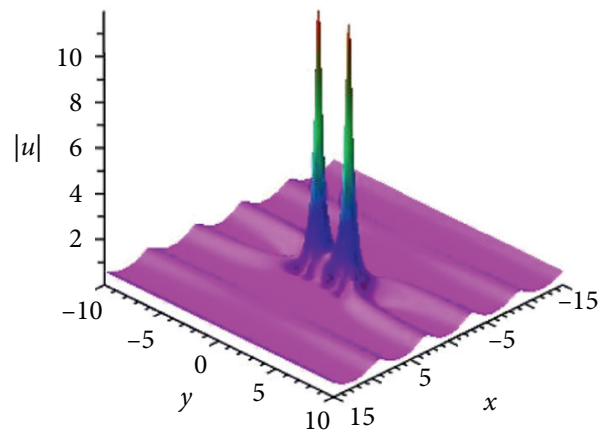

(c)

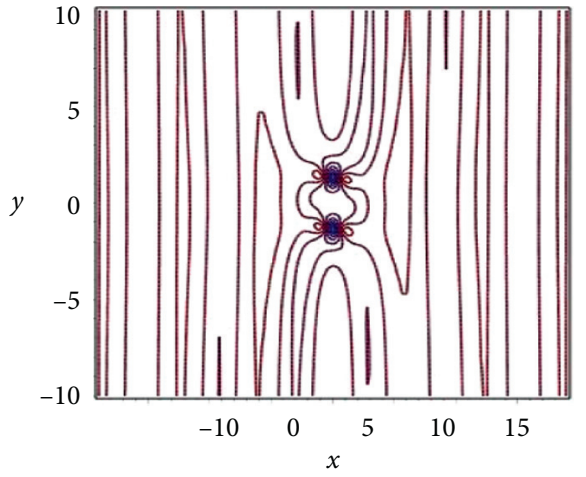

(b)

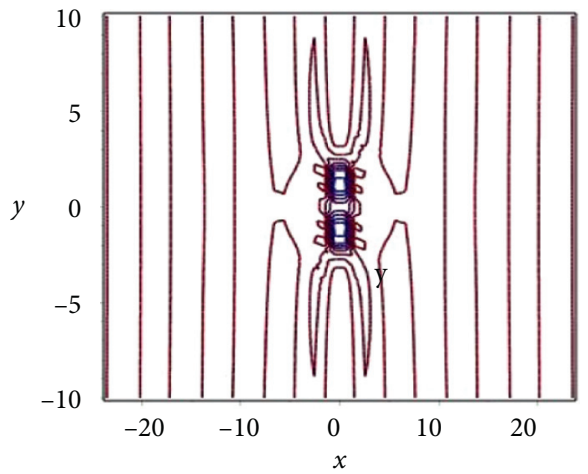

(d)

FIGURE 9: Semirational solutions constituting of two lumps and periodic line waves for the nonlocal Mel'nikov equation with parameters $\kappa=1, \lambda_{1}=-\lambda_{2}=1, \lambda_{3}=-\lambda_{4}=2, P_{5}=1, Q_{5}=0$, and $\eta_{5}^{0}=-(\pi / 6)$. The panels (b) and (d) are the contour plots of the panels (a) and (c), respectively.

The second bilinear form of equation (60) not only defines the amplitude parameter $A_{0}$ but also imposes additional constraints in terms of the theta constants:

$$
\begin{aligned}
& \alpha^{2}\left[\theta_{3}^{4}(0, \tau)+\theta_{4}^{4}(0, \tau)\right]+\alpha^{4} \frac{\theta_{3}^{\prime \prime \prime}(0, \tau) \theta_{4}(0, \tau)+\theta_{4}^{\prime \prime \prime \prime}(0, \tau) \theta_{3}(0, \tau)+6 \theta_{3}^{\prime \prime}(0, \tau) \theta_{4}^{\prime \prime}(0, \tau)}{\theta_{3}(0, \tau) \theta_{4}(0, \tau)} \\
& -\alpha^{4} \frac{\left[2 \theta_{2}^{\prime \prime \prime}(0, \tau) \theta_{2}(0, \tau)+6\left(\theta_{2}^{\prime \prime}(0, \tau)\right)^{2}\right]}{\left(\theta_{2}(0, \tau)\right)^{2}}-3 \omega^{2}\left[\theta_{3}^{4}\left(0, \tau_{1}\right)+\theta_{4}^{4}\left(0, \tau_{1}\right)\right] \\
& =\sigma A_{0}^{2} \frac{\theta_{3}^{2}\left(0, \tau_{1}\right)}{\theta_{2}^{2}\left(0, \tau_{1}\right)} \\
& 2 \alpha^{2} \theta_{3}^{2}(0, \tau) \theta_{4}^{2}(0, \tau)+8 \alpha^{4} \frac{\theta_{1}^{\prime}(0, \tau) \theta_{1}^{\prime \prime \prime}(0, \tau)}{\theta_{2}^{2}(0, \tau)}-6 \omega^{2} \theta_{3}^{2}\left(0, \tau_{1}\right) \theta_{4}^{2}\left(0, \tau_{1}\right) \\
& =\sigma A_{0}^{2} \frac{\theta_{4}^{2}\left(0, \tau_{1}\right)}{\theta_{2}^{2}\left(0, \tau_{1}\right)} .
\end{aligned}
$$

The three constraints equations (63)-(65) then determine the parameter $A_{0}^{2}, \tau$, and $\tau_{1}$, assuming $\sigma$ and $\alpha$ are given (and $\omega$ through equation (62)). The solution equation (61b) can be expressed in terms of Jacobi elliptic function in a slightly more condensed but less symmetric forms, with again different $k$ and $k_{1}$ : 


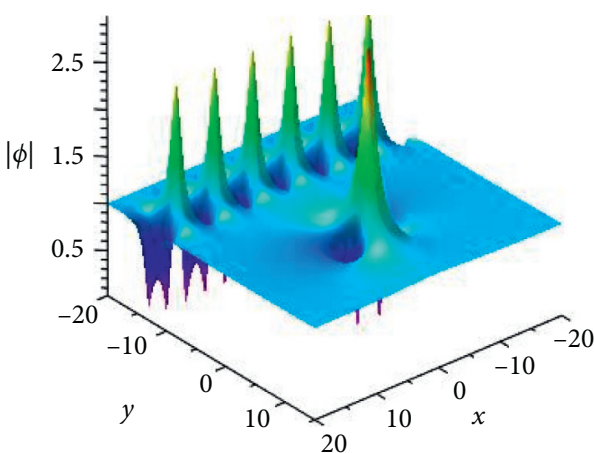

(a)

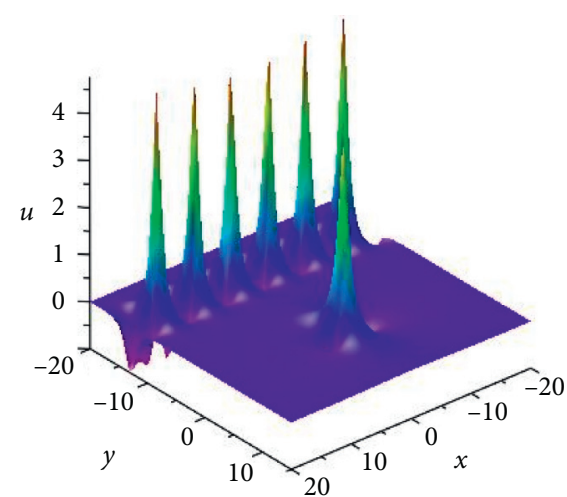

(b)

FIGURE 10: Semirational solutions $|\phi|$ and $u$ consisting of a lump and one breather for the nonlocal Mel'nikov equation with parameters $\kappa=1, \lambda_{1}=1, \lambda_{2}=-1, P_{3}=-P_{4}=1, Q_{3}=Q_{4}=1, \eta_{3}^{0}=\eta_{4}^{0}=-2 \pi$, and $t=0$.

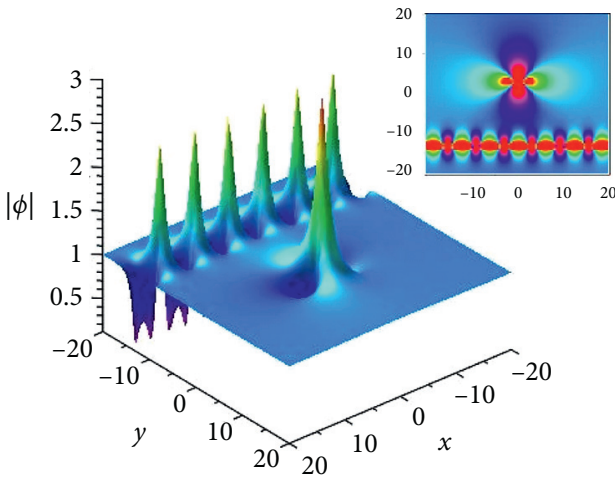

(a)

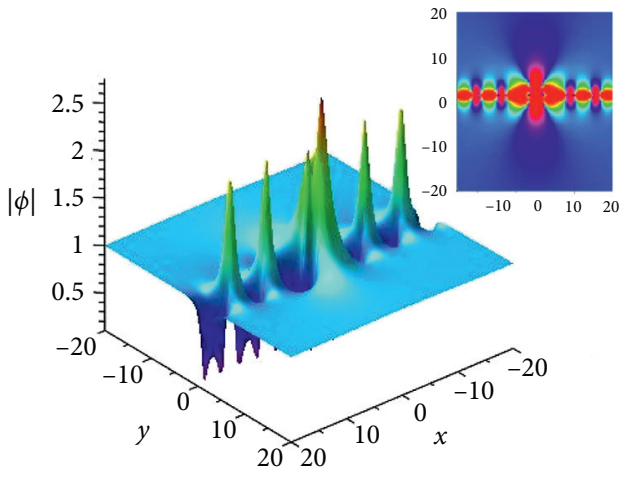

(c)

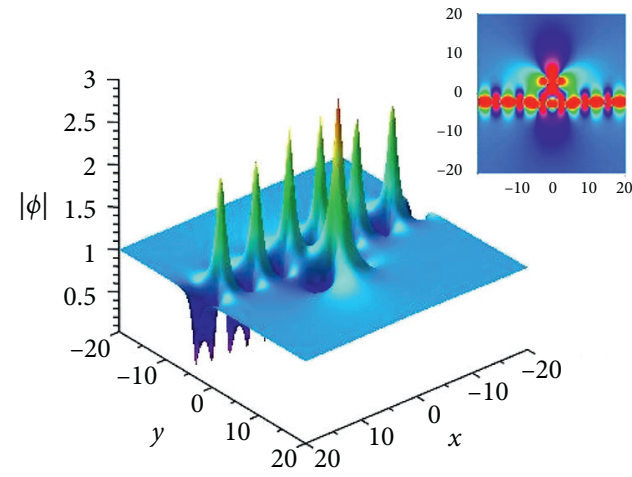

(b)

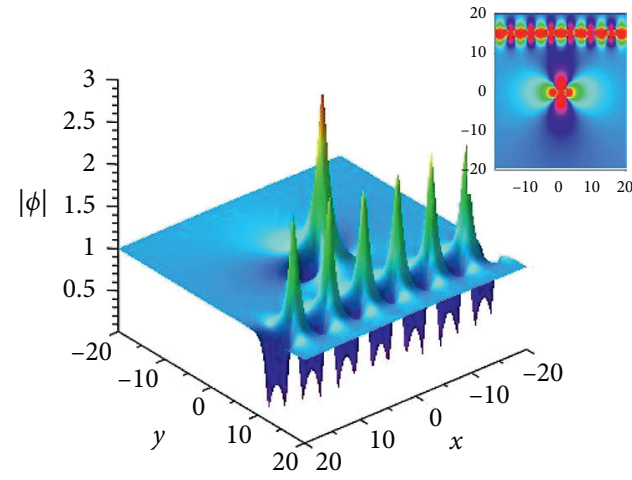

(d)

Figure 11: The superposition of a lump and one breather with parameters $\kappa=1, \lambda_{1}=1, \lambda_{2}=-1, P_{3}=-P_{4}=1, Q_{3}=Q_{4}=1$, and $t=0$ for different values of $\eta_{3}^{0}$. (a) $\eta_{3}^{0}=-2 \pi$. (b) $\eta_{3}^{0}=0$. (c) $\eta_{3}^{0}=\pi$. (d) $\eta_{3}^{0}=2 \pi$.

$$
\begin{aligned}
A & =A_{0} \sqrt{k_{1}} \frac{\mathrm{cn}\left(s t, k_{1}\right)+i \sqrt{1+k_{1}} \operatorname{sn}\left(s t, k_{1}\right) \operatorname{dn}(r x, k)}{\sqrt{1+k_{1}} \operatorname{dn}(r x, k)+\operatorname{dn}\left(s t, k_{1}\right)} \exp (-i \Omega t), \\
s & =\frac{2 r^{2}}{1+k_{1}}, \\
k^{2} & =\frac{2 k_{1}}{1+k_{1}} .
\end{aligned}
$$

The advantage is that most computer algebra software can handle Jacobi elliptic functions efficiently.

\section{Discussion and and Conclusions}

In this paper, we have introduced and investigated a nonlocal $\mathrm{Mel}^{\prime}$ nikov equation with partial reverse space time, which constitutes a multidimensional version of the nonlocal Schrödinger-Boussinesq equation with a parity-time- 
symmetric potential. By using the Hirota bilinear method, soliton solutions are obtained. Although these soliton solutions may have singularities, general $n$-breather solutions and mixed solutions consisting of breathers and periodic line waves can be derived under proper parameter constraints and lead to nonsingular solutions. On taking a long wave limit, nonsingular rational solutions or lumps can be generated. The exact explicit lump solutions up to the third order are presented, and properties of the dynamics of interaction between lumps have been revealed. Furthermore, by taking a coalescence of wavenumbers at a finite value, two subclasses of semirational solutions are derived. One subclass of these semirational solutions describes lumps on a background of periodic line waves. Another one highlights interaction between lumps and breathers. In particular, two solutions of the nonlocal Schrödinger-Boussinesq equation, namely, fundamental rogue waves and mixed modes consisting of a rogue wave and periodic line waves, are obtained as reductions of the corresponding solutions of the partial reverse space-time nonlocal $\mathrm{Mel}^{\prime}$ nikov equation. Finally, special classes of doubly periodic solutions are presented too. We believe that similar techniques can be applied to many nonlocal evolution equations being studied in the literature. As nonlocal differential equations have been amply demonstrated in the literature to be applicable to many fields, e.g., population dynamics [25] and microelectro mechanical system [63], further efforts will likely be fruitful.

\section{Appendix}

\section{A: Higher oder partial solutions}

The functions $f_{6}$ and $g_{6}^{0}$ read as

$$
\begin{aligned}
& f_{6}=y^{6}+\left(\frac{29 x^{2}}{4}-\frac{173731727}{176400}-\frac{425 t x}{4}+\frac{6525 t^{2}}{16}\right) y^{4} \\
& +\left(\frac{61 x^{4}}{4}-\frac{1685 t x^{3}}{4}+\left(\frac{34675 t^{2}}{8}+\frac{291073289}{176400}\right) x^{2}-\frac{314125 t^{3} x}{16}\right. \\
& \left.+\frac{503447329781761}{1944810000}+\frac{870750371 t^{2}}{9408}+\frac{2113125 t^{4}}{64}\right) y^{2} \\
& +9 x^{6}-\frac{705 x^{5} t}{2}+\left(\frac{18149933}{4900}+\frac{90925 t^{2}}{16}\right) x^{4}-\frac{772625 t^{3} x^{3}}{16} \\
& +\left(\frac{24747767333 t^{2}}{28224}+\frac{19715017078429}{54022500}+\frac{14608125 t^{4}}{64}\right) x^{2} \\
& +\left(-\frac{231250057 t y^{2}}{8820}-\frac{7239053785 t^{3}}{2016}-\frac{18221875 t^{5}}{32}\right) x+\frac{37515625 t^{6}}{64} \\
& +\frac{1569350425 t^{4}}{288}+\frac{742769909281}{1500625}+\frac{3798316849584289 t^{2}}{311169600} \\
& -\frac{273805647604109 t x}{64827000}-\frac{551823397 t x^{3}}{5880} \\
& g_{6}^{0}=A_{6}+i B_{6} \\
& A_{6}=-261 x^{4}+\frac{12795}{2} t x^{3}+\left(-\frac{944525 t^{2}}{16}-\frac{3065 y^{2}}{4}+\frac{16054106}{1225}\right) x^{2} \\
& +\left(\frac{1936375 t^{3}}{8}+9965 t y^{2}-\frac{70472141 t}{735}\right) x+\left(-\frac{2970625 t^{4}}{8}-\frac{548225 t^{2} y^{2}}{16}+\frac{617733841 t^{2}}{3528}-\frac{1329 y^{4}}{4}\right. \\
& \left.+\frac{2329695913 y^{2}}{22050}-\frac{3199122765724}{1500625}\right) \\
& B_{6}=y\left[108 x^{4}-2820 t x^{3}+\left(122 y^{2}+\frac{55225}{2} t^{2}-1566\right) x^{2}+\left(-\frac{478625 t^{3}}{4}-1685 t y^{2}-\frac{20569361 t x}{1470}\right) x\right. \\
& \left.+\frac{3093125 t^{4}}{16}+\frac{12275 t^{2} y^{2}}{2}+\frac{691962241 t^{2}}{7056}+29 y^{4}-\frac{167205151 y^{2}}{8820}+\frac{22387448589829}{13505625}\right] \text {. }
\end{aligned}
$$


The functions $f$ and $g$ generated from 5-soliton solution of the nonlocal Mel'nikov equation are defined as

$$
\begin{aligned}
& f=\left(\theta_{1} \theta_{2} \theta_{3} \theta_{4}+a_{12} \theta_{3} \theta_{4}+a_{13} \theta_{2} \theta_{4}+a_{14} \theta_{2} \theta_{3}+a_{23} \theta_{1} \theta_{4}+a_{24} \theta_{1} \theta_{3}+a_{34} \theta_{1} \theta_{2}+a_{12} a_{34}+a_{13} a_{24}+a_{14} a_{23}\right) \\
& +e^{\eta_{5}}\left[\theta_{1} \theta_{2} \theta_{3} \theta_{4}+a_{45} \theta_{1} \theta_{2} \theta_{3}+a_{35} \theta_{1} \theta_{2} \theta_{4}+a_{25} \theta_{1} \theta_{3} \theta_{4}+a_{15} \theta_{2} \theta_{3} \theta_{4}+\left(a_{35} a_{45}+a_{34}\right) \theta_{1} \theta_{2}+\left(a_{25} a_{45}+a_{24}\right) \theta_{1} \theta_{3}\right. \\
& +\left(a_{25} a_{35}+a_{23}\right) \theta_{1} \theta_{4}+\left(a_{15} a_{45}+a_{14}\right) \theta_{2} \theta_{3}+\left(a_{15} a_{35}+a_{13}\right) \theta_{2} \theta_{4}+\left(a_{15} a_{25}+a_{12}\right) \theta_{3} \theta_{4} \\
& +\left(a_{25} a_{35} a_{45}+a_{23} a_{45}+a_{25} a_{34}+a_{24} a_{35}\right) \theta_{1}+\left(a_{15} a_{35} a_{45}+a_{14} a_{35}+a_{13} a_{45}+a_{15} a_{34} \theta_{2}\right) \\
& +\left(a_{15} a_{25} a_{45}+a_{14} a_{25}+a_{15} a_{24}+a_{12} a_{45}\right) \theta_{3}+\left(a_{15} a_{25} a_{35}+a_{15} a_{23}+a_{13} a_{25}+a_{12} a_{35}\right) \theta_{4} \\
& \left.+a_{12} a_{34}+a_{13} a_{24}+a_{14} a_{23}+a_{12} a_{35} a_{45}+a_{13} a_{25} a_{45}+a_{14} a_{25} a_{35}+a_{15} a_{24} a_{35}+a_{15} a_{25} a_{34}+a_{15} a_{23} a_{45}+a_{15} a_{25} a_{35} a_{45}\right] \text {, } \\
& g=\left[\left(\theta_{1}+b_{1}\right)\left(\theta_{2}+b_{2}\right)\left(\theta_{3}+b_{3}\right)\left(\theta_{4}+b_{4}\right)+a_{12}\left(\theta_{3}+b_{3}\right)\left(\theta_{4}+b_{4}\right)+a_{13}\left(\theta_{2}+b_{2}\right)\left(\theta_{4}+b_{4}\right)+a_{14}\left(\theta_{2}+b_{2}\right)\left(\theta_{3}+b_{3}\right)\right. \\
& \left.+a_{23}\left(\theta_{1}+b_{1}\right)\left(\theta_{4}+b_{4}\right) a_{24}\left(\theta_{1}+b_{1}\right)\left(\theta_{3}+b_{3}\right)+a_{34}\left(\theta_{1}+b_{1}\right)\left(\theta_{2}+b_{2}\right)+a_{12} a_{34}+a_{13} a_{24}+a_{14} a_{23}\right] \\
& +e^{\eta_{5}+i \phi_{5}}\left[\left(\theta_{1}+b_{1}\right)\left(\theta_{2}+b_{2}\right)\left(\theta_{3}+b_{3}\right)\left(\theta_{4}+b_{4}\right)+a_{45}\left(\theta_{1}+b_{1}\right)\left(\theta_{2}+b_{2}\right)\left(\theta_{3}+b_{3}\right)\right. \\
& +a_{35}\left(\theta_{1}+b_{1}\right)\left(\theta_{2}+b_{2}\right)\left(\theta_{4}+b_{4}\right)+a_{25}\left(\theta_{1}+b_{1}\right)\left(\theta_{3}+b_{3}\right)\left(\theta_{4}+b_{4}\right)+a_{15}\left(\theta_{2}+b_{2}\right)\left(\theta_{3}+b_{3}\right)\left(\theta_{4}+b_{4}\right) \\
& +\left(a_{35} a_{45}+a_{34}\right)\left(\theta_{1}+b_{1}\right)\left(\theta_{2}+b_{2}\right)+\left(a_{25} a_{45}+a_{24}\right)\left(\theta_{1}+b_{1}\right)\left(\theta_{3}+b_{3}\right)+\left(a_{25} a_{35}+a_{23}\right)\left(\theta_{2}+b_{2}\right)\left(\theta_{4}+b_{4}\right) \\
& +\left(a_{15} a_{45}+a_{14}\right)\left(\theta_{2}+b_{2}\right)\left(\theta_{3}+b_{3}\right)+\left(a_{15} a_{35}+a_{13}\right)\left(\theta_{2}+b_{2}\right)\left(\theta_{4}+b_{4}\right)+\left(a_{15} a_{25}+a_{12}\right)\left(\theta_{3}+b_{3}\right)\left(\theta_{4}+b_{4}\right) \\
& +\left(a_{25} a_{35} a_{45}+a_{23} a_{45}\left(\theta_{2}+b_{2}\right)+\left(a_{15} a_{25} a_{45}+a_{14} a_{25}+a_{15} a_{24}+a_{12} a_{45}\right)\left(\theta_{3}+b_{3}\right)\right. \\
& +\left(a_{15} a_{25} a_{35}+a_{15} a_{23}+a_{13} a_{25}+a_{12} a_{35}\right)\left(\theta_{4}+b_{4}\right)+a_{12}\left(a_{34}+a_{35} a_{45}\right)+a_{13}\left(a_{24}+a_{25} a_{45}\right) \\
& \left.+a_{14}\left(a_{23}+a_{25} a_{35}\right)+a_{15}\left(a_{24} a_{35}+a_{25} a_{34}+a_{23} a_{45}+a_{25} a_{35} a_{45}\right)\right] \text {, }
\end{aligned}
$$

where

$$
a_{j 5}=-\frac{4 P_{5}^{2}}{P_{5}^{2}+\lambda_{j}^{2}}
$$

and $\theta_{j}, b_{j}, a_{i j}(1 \leq i<j \leq 4)$ are given by equation (30); $\eta_{5}$ and $\phi_{5}$ are given by equation (14).

The functions $f$ and $g$ generated from 4-soliton solution of the $\mathrm{Mel}^{\prime}$ nikov equation are written as

$$
\begin{aligned}
f= & e^{A_{34}}\left(a_{13} a_{23}+a_{13} a_{24}+a_{13} \theta_{2}+a_{14} a_{23}+a_{14} a_{24}+a_{14} \theta_{2}+a_{23} \theta_{1}+a_{24} \theta_{1}+\theta_{1} \theta_{2}+a_{12} e^{\eta_{3}+\eta_{4}}\right) \\
& +\left(a_{13} a_{23}+a_{13} \theta_{2}+a_{23} \theta_{1}+\theta_{1} \theta_{2}+a_{12}\right) e^{\eta_{3}} \\
& +\left(a_{14} a_{24}+a_{14} \theta_{2}+a_{24} \theta_{1}+\theta_{1} \theta_{2}+a_{12}\right) e^{\eta_{4}}+\theta_{1} \theta_{2}+a_{12}, \\
g= & e^{A_{34}}\left[a_{13} a_{23}+a_{13} a_{24}+a_{13}\left(\theta_{2}+b_{2}\right)+a_{14} a_{23}+a_{14} a_{24}+a_{14}\left(\theta_{2}+b_{2}\right)+a_{23}\left(\theta_{1}+b_{1}\right)+a_{24}\left(\theta_{1}+b_{1}\right)\right. \\
& \left.+\left(\theta_{1}+b_{1}\right)\left(\theta_{2}+b_{2}\right)+a_{12}\right] e^{\eta_{3}+i \phi_{3}+\eta_{4}+i \phi_{4}} \\
& +\left[a_{13} a_{23}+a_{13}\left(\theta_{2}+b_{2}\right)+a_{23}\left(\theta_{1}+b_{1}\right)+\left(\theta_{1}+b_{1}\right)\left(\theta_{2}+b_{2}\right)+a_{12}\right] e^{\eta_{3}+i \phi_{3}} \\
& +\left[a_{14} a_{24}+a_{14}\left(\theta_{2}+b_{2}\right)+a_{24}\left(\theta_{1}+b_{1}\right)+\left(\theta_{1}+b_{1}\right)\left(\theta_{2}+b_{2}\right)+a_{12}\right] e^{\eta_{4}+i \phi_{4}} \\
& +\left(\theta_{1}+b_{1}\right)\left(\theta_{2}+b_{2}\right)+a_{12},
\end{aligned}
$$

where

$$
a_{s l}=\frac{4 P_{l}^{3}}{P_{l}^{4}+\left(\lambda_{2} P_{l}-Q_{l}\right)^{2}} \quad(s=1,2, l=3,4),
$$


and $a_{12}, b_{s}, \phi_{l}, \eta_{l}$, and $e^{A_{34}}$ are given by (30) and (14).

\section{B: Elliptic and theta functions}

The four theta functions $\theta_{n}(x, \tau)(n=1,2,3,4)$ and the modulus parameters $q$ (the nome) and $\tau$ (pure imaginary) are given by

$$
\begin{aligned}
& \theta_{1}(x, \tau)=2 \sum_{n=0}^{\infty}(-1)^{n} q^{(n+(1 / 2))^{2}} \sin (2 n+1) x \\
& =-\sum_{n=-\infty}^{\infty} \exp \left(\pi i \tau(m+(1 / 2))^{2}\right. \\
& \left.+2 i\left(m+\frac{1}{2}\right)\left(x+\frac{\pi}{2}\right)\right), \\
& \theta_{2}(x, \tau)=2 \sum_{n=0}^{\infty} q^{(n+(1 / 2))^{2}} \cos (2 n+1) x \\
& =\sum_{n=-\infty}^{\infty} \exp \left(\pi i \tau\left(m+\frac{1}{2}\right)^{2}+2 i\left(m+\frac{1}{2}\right) x\right), \\
& \theta_{3}(x, \tau)=1+2 \sum_{n=1}^{\infty} q^{n^{2}} \cos (2 n x) \\
& =\sum_{n=-\infty}^{\infty} \exp \left(\pi i \tau m^{2}+2 i m\left(x+\frac{\pi}{2}\right)\right), \\
& \theta_{4}(x, \tau)=1+2 \sum_{n=1}^{\infty}(-1)^{n} q^{n^{2}} \cos (2 n x) \\
& =\sum_{n=-\infty}^{\infty} \exp \left(\pi i \tau m^{2}+2 i m\left(x+\frac{\pi}{2}\right)\right), \\
& 0<q<1, \quad q=\exp (\pi i \tau)=\exp \left(-\frac{\pi K^{\prime}}{K}\right)
\end{aligned}
$$

$K$ and $K^{\prime}$ are the complete elliptic integrals of the first kind with parameters $k$ and $\left(1-k^{2}\right)^{1 / 2} \cdot \theta_{1}$ is odd while the other three are even. The zeros of $\theta_{1}, \theta_{2}, \theta_{3}$, and $\theta_{4}$ are at $M \pi+$ $N \pi \tau,(M+(1 / 2)) \pi+N \pi \tau,(M+(1 / 2)) \pi+(N+(1 / 2)) \pi \tau$, $M \pi+(N+(1 / 2)) \pi \tau$, respectively, $(M, N$ integers). The pairs $\left(\theta_{1}, \theta_{2}\right),\left(\theta_{3}, \theta_{4}\right)$ are related by phase shift of $\pi / 2$. The dependence on the parameter may be dropped for simplicity, and we shall just write $\theta_{n}(x, \tau) \equiv \theta_{n}(x)$. Theta and elliptic functions are related by

$$
\begin{aligned}
\operatorname{sn}(u) & =\frac{\theta_{3}(0) \theta_{1}(z)}{\theta_{2}(0) \theta_{4}(z)}, \\
\operatorname{cn}(u) & =\frac{\theta_{4}(0) \theta_{2}(z)}{\theta_{2}(0) \theta_{4}(z)}, \\
\operatorname{dn}(u) & =\frac{\theta_{4}(0) \theta_{3}(z)}{\theta_{3}(0) \theta_{4}(z)}, \\
z & =\frac{u}{\theta_{3}^{2}(0)}, \\
k & =\frac{\theta_{2}^{2}(0)}{\theta_{3}^{2}(0)}, \\
k^{\prime} & =\frac{\theta_{4}^{2}(0)}{\theta_{3}^{2}(0)}, \\
k^{2}+\left(k^{\prime}\right)^{2} & =1 .
\end{aligned}
$$

Theta functions possess a huge number of identities, e.g., $\theta_{3}(x+y) \theta_{3}(x-y) \theta_{2}^{2}(0)=\theta_{4}^{2}(x) \theta_{1}^{2}(y)+\theta_{3}^{2}(x) \theta_{2}^{2}(y)$,

$$
\theta_{4}(x+y) \theta_{4}(x-y) \theta_{2}^{2}(0)=\theta_{4}^{2}(x) \theta_{2}^{2}(y)+\theta_{3}^{2}(x) \theta_{1}^{2}(y) .
$$

On differentiating equations (B.3a) and (B.3b) with respect to $y$, setting $y=0$ will yield

$$
\begin{aligned}
& D_{x}^{2} \theta_{3}(x) \cdot \theta_{3}(x)=\frac{2 \theta_{2}^{\prime \prime}(0)_{3}^{2}(x)}{\theta_{2}(0)}+2 \theta_{3}^{2}(0) \theta_{4}^{2}(0) \theta_{4}^{2}(x), \\
& D_{x}^{2} \theta_{4}(x) \cdot \theta_{4}(x)=2 \theta_{3}^{2}(0) \theta_{4}^{2}(0) \theta_{3}^{2}(x)+\frac{2 \theta_{2}^{\prime \prime}(0) \theta_{4}^{2}(x)}{\theta_{2}(0)},
\end{aligned}
$$

by using $\theta_{1}^{\prime}(0)=\theta_{2}(0) \theta_{3}(0) \theta_{4}(0)$. Hence, formulae for $D_{x} \theta_{m} \cdot \theta_{n}, D_{x}^{2} \theta_{m} \cdot \theta_{n}$ can be developed for $m$ and $n$ integers using this line of reasoning.

\section{Data Availability}

The data used to support the findings of this study are included within the article.

\section{Conflicts of Interest}

The authors declare that they have no conflicts of interest.

\section{Acknowledgments}

This work was sponsored by the National Natural Science Foundation of China (no. 11571079, 11975131 and 11801321), Shanghai Pujiang Program (no. 14PJD007), Natural Science Foundation of Shanghai (no. 14ZR1403500), and Young Teachers Foundation (no. 1411018) of Fudan University. WL wishes to thank Prof Jingsong He of Ningbo 
University and Dr Jiguang Rao of University of Science and Technology of China for their many discussions and suggestions on the paper. Partial support for KWC has been provided by the Research Grants Council General Research Fund Contract (no. HKU 17200815 and 17200718).

\section{References}

[1] C. Hamner, J. J. Chang, P. Engels, and M. A. Hoefer, "Generation of dark-bright soliton trains in superfluid-superfluid counterflow," Physical Review Letters, vol. 106, no. 6, Article ID 065302, 2011.

[2] L. Wang, J. H. Zhang, C. Liu, M. Li, and F. H. Qi, "Breather transition dynamics, Peregrine combs and walls, and modulation instability in a variable-coefficient nonlinear Schrödinger equation with higher-order effects," Physical Review E, vol. 93, no. 6, Article ID 062217, 2016.

[3] L. Wang, J. H. Zhang, Z. Q. Wang et al., "Breather-to-soliton transitions, nonlinear wave interactions, and modulational instability in a higher-order generalized nonlinear Schrödinger equation," Physical Review E, vol. 93, no. 1, Article ID 012214, 2016.

[4] L. Wang, M. Li, F. H. Qi, and T. Xu, "Modulational instability, nonautonomous breathers and rogue waves for a variablecoefficient derivative nonlinear Schrödinger equation in the inhomogeneous plasmas," Physics of Plasmas, vol. 22, no. 3, p. 520, 2015.

[5] D. Mihalache, "Multidimensional localized structures in optics and Bose-Einstein condensates: a selection of recent studies," Rom. J. Phys.vol. 59, no. 3, pp. 295-312, 2014.

[6] V. S. Bagnato, D. J. Frantzeskakis, P. G. Kevrekidis, B. A. Malomed, and D. Mihalache, "Comments: special volume of Romanian reports in physics dedicated to BoseEinstein condensation," Romanian Reports in Physics, vol. 67, pp. 5-50, 2015.

[7] B. Malomed, L. Torner, F. Wise, and D. Mihalache, "On multidimensional solitons and their legacy in contemporary atomic, molecular and optical physics," Journal of Physics B: Atomic, Molecular and Optical Physics, vol. 49, no. 17, p. 170502, 2016.

[8] P. G. Kevrekidis and D. J. Frantzeskakis, "Solitons in coupled nonlinear Schrödinger models: a survey of recent developments," Reviews in Physics, vol. 1, pp. 140-153, 2016.

[9] V. B. Matveev and A. M. Salle, Darboux Transformation and Solitons, Springer, Berlin, Germany, 1991.

[10] C. H. Gu, H. S. Hu, and Z. X. Zhou, Darboux Transformation in Soliton Theory and Geometric Applications, Shanghai Science and Technology Press, Shanghai, China, 1999.

[11] R. Hirota, The Direct Method in Soliton Theory, Cambridge University Press, Cambridge, UK, 2004.

[12] V. A. Vladimirov and C. Mączka, "Exact solutions of generalized Burgers equation, describing travelling fronts and their interaction," Reports on Mathematical Physics, vol. 60, no. 2, pp. 317-328, 2007.

[13] M. T. Darvishi, M. Najafi, L. Kavitha, and M. Venkatesh, "Stair and step soliton solutions of the integrable $(2+1)$ and $(3$ + 1)-dimensional Boiti-Leon-Manna-Pempinelli equations," Communications in Theoretical Physics, vol. 58, no. 6, pp. 785-794, 2012.

[14] M. J. Ablowitz, Solitons, Nonlinear Evolution Equations and Inverse Scattering, Cambridge University Press, Cambridge, UK, 1992.

[15] L. A. Dickey, Soliton Equations and Hamiltonian Systems, World Scientific, Singapore, 2003.
[16] M. Jimbo and T. Miwa, "Solitons and infinite-dimensional Lie algebras," Publications of the Research Institute for Mathematical Sciences, vol. 19, no. 3, pp. 943-1001, 1983.

[17] Z. H. Zhao and Z. D. Dai, "Explicit non-travelling wave solutions for non-integrable $(3+1)$-dimensional systems," International Journal of Nonlinear Sciences and Numerical Simulation, vol. 11, pp. 679-688, 2010.

[18] W. X. Ma, "Lump solutions to the Kadomtsev-Petviashvili equation," Physics Letters A, vol. 379, no. 36, pp. 1975-1978, 2015.

[19] W. X. Ma, Z. Qin, and X. Lü, "Lump solutions to dimensionally reduced -gKP and -gBKP equations," Nonlinear Dynamics, vol. 84, no. 2, pp. 923-931, 2016.

[20] S. Kheybari, M. T. Darvishi, and A. M. Wazwaz, "A semianalytical algorithm to solve systems of integro-differential equations under mixed boundary conditions," Journal of Computational and Applied Mathematics, vol. 317, pp. 72-89, 2017.

[21] G. Bruell, M. Ehrnström, and L. Pei, "Symmetry and decay of traveling wave solutions to the Whitham equation," Journal of Differential Equations, vol. 262, no. 8, pp. 4232-4254, 2017.

[22] J. Li, E. Latos, and L. Chen, "Wavefronts for a nonlinear nonlocal bistable reaction-diffusion equation in population dynamics," Journal of Differential Equations, vol. 263, no. 10, pp. 6427-6455, 2017.

[23] K. Kuto, T. Mori, T. Tsujikawa, and S. Yotsutani, "Global solution branches for a nonlocal Allen-Cahn equation," Journal of Differential Equations, vol. 264, no. 9, pp. 59285949, 2018.

[24] M. J. Ablowitz and Z. H. Musslimani, "Integrable nonlocal nonlinear Schrödinger equation," Physical Review Letters, vol. 110, Article ID 064105, 2013.

[25] A. S. Fokas, "Integrable multidimensional versions of the nonlocal nonlinear Schrödinger equation," Nonlinearity, vol. 29, no. 2, pp. 319-324, 2016.

[26] M. J. Ablowitz and Z. H. Musslimani, "Integrable discrete PT symmetric model," Physical Review E, vol. 90, Article ID 032912, 2014.

[27] Z. Yan, "Integrable PT-symmetric local and nonlocal vector nonlinear Schrödinger equations: a unified two-parameter model," Applied Mathematics Letters, vol. 47, pp. 61-68, 2015.

[28] A. Khara and A. Saxena, "Periodic and hyperbolic soliton solutions of a number of nonlocal nonlinear equations," Journal of Mathematical Physics, vol. 56, no. 3, Article ID 032104, 2015.

[29] M. J. Ablowitz and Z. H. Musslimani, "Inverse scattering transform for the integrable nonlocal nonlinear Schrödinger equation," Nonlinearity, vol. 29, no. 3, pp. 915-946, 2016.

[30] C. Q. Song, D. M. Xiao, and Z. N. Zhu, "Solitons and dynamics for a general integrable nonlocal coupled nonlinear Schrödinger equation," Communications in Nonlinear Science and Numerical Simulation, vol. 45, pp. 13-28, 2017.

[31] S. Y. Lou, "Alice-Bob systems, Ps-Td-C principles and multisoliton solutions," Journal of Mathematical Physics, vol. 59, no. 8, Article ID 083507, 2018.

[32] S. Y. Lou and F. Huang, "Alice-Bob physics: coherent solutions of nonlocal KdV systems," Scientific Reports, vol. 7, no. 1, p. 869, 2017.

[33] M. J. Ablowitz and Z. H. Musslimani, "Integrable nonlocal nonlinear equations," Studies in Applied Mathematics, vol. 139, no. 1, pp. 7-59, 2016.

[34] Z. X. Xu and K. W. Chow, "Breathers and rogue waves for a third order nonlocal partial differential equation by a bilinear 
transformation," Applied Mathematics Letters, vol. 56, pp. 72-77, 2016.

[35] Z. X. Zhou, "Darboux transformations and global solutions for a nonlocal derivative nonlinear Schrödinger equation," Communications in Nonlinear Science and Numerical Simulation, vol. 62, pp. 480-488, 2018.

[36] Z. X. Zhou, "Darboux transformations and global explicit solutions for nonlocal Davey-Stewartson I equation," Studies in Applied Mathematics, vol. 141, no. 2, pp. 186-204, 2018.

[37] J. G. Rao, Y. S. Zhang, A. S. Fokas, and J. S. He, "Rogue waves of the nonlocal Davey-Stewartson I equation," Nonlinearity, vol. 31, no. 9, pp. 4090-4107, 2018.

[38] J. Rao, Y. Cheng, and J. He, "Rational and semirational solutions of the nonlocal davey-stewartson equations," Studies in Applied Mathematics, vol. 139, no. 4, pp. 568-598, 2017.

[39] J. L. Ji and Z. N. Zhu, "On a nonlocal modified Korteweg-de Vries equation: integrability, Darboux transformation and soliton solutions," Communications in Nonlinear Science and Numerical Simulation, vol. 42, pp. 699-708, 2017.

[40] L. Y. Ma, S. F. Shen, and Z. N. Zhu, "Integrable nonlocal complex mKdV equation: soliton solution and gauge equivalence," Journal of Mathematical Physics, vol. 58, p. 103501, 2018.

[41] V. S. Gerdjikov and A. Saxena, "Complete integrability of nonlocal nonlinear Schrödinger equation," Journal of Mathematical Physics, vol. 58, no. 1, Article ID 013502, 2017.

[42] J. Rao, Y. Cheng, K. Porsezian, D. Mihalache, and J. He, "PTsymmetric nonlocal Davey-Stewartson I equation: soliton solutions with nonzero background," Physica D: Nonlinear Phenomena, vol. 401, p. 132180, 2020.

[43] M. J. Ablowitz, X.-D. Luo, and Z. H. Musslimani, "Inverse scattering transform for the nonlocal nonlinear Schrödinger equation with nonzero boundary conditions," Journal of Mathematical Physics, vol. 59, no. 1, Article ID 011501, 2018.

[44] V. K. Mel'nikov, "On equations for wave interactions," Letters in Mathematical Physics, vol. 7, pp. 129-136, 1983.

[45] V. K. Mel'nikov, "Wave emission and absorption in a nonlinear integrable system," Physics Letters A, vol. 118, no. 1, pp. 22-24, 1986.

[46] V. K. Mel'nikov, "Reflection of waves in nonlinear integrable systems," Journal of Mathematical Physics, vol. 28, no. 11, pp. 2603-2609, 1987.

[47] V. K. Mel'nikov, "A direct method for deriving a multi-soliton solution for the problem of interaction of waves on the $x, y$ plane," Communications in Mathematical Physics, vol. 112, no. 4, pp. 639-652, 1987.

[48] C. S. Kumar, R. Radha, and M. Lakshmanan, "Exponentially localized solutions of Mel'nikov equation," Chaos, Solitons \& Fractals, vol. 22, no. 3, pp. 705-712, 2004.

[49] Y. Hase, R. Hirota, Y. Ohta, and J. Satsuma, "Soliton solutions to the Mel'nikov equations," Journal of the Physical Society of Japan, vol. 58, no. 8, pp. 2713-2720, 1989.

[50] G. Mu and Z. Qin, "Two spatial dimensional N-rogue waves and their dynamics in Mel'nikov equation," Nonlinear Analysis: Real World Applications, vol. 18, pp. 1-13, 2014.

[51] Z. Han, Y. Chen, and J. C. Chen, "General N-dark soliton solutions of the multi-component Mel'nikov system," Journal of the Physical Society of Japan, vol. 86, no. 7, Article ID 074005, 2017.

[52] G. Mu and Z. Y. Qin, "Rogue waves for the coupled Schrödinger-Boussinesq equation and the coupled Higgs equation," Journal of the Physical Society of Japan, vol. 81, no. 8, Article ID 084001, 2012.
[53] J. S. He, L. H. Wang, L. J. Li, K. Porsezian, and R. Erdélyi, "Few-cycle optical rogue waves: complex modified Korteweg de Vries equation," Physical Review E, vol. 89, no. 6, Article ID 062917, 2014

[54] S. W. Xu, J. S. He, K. Porsezian, and Y. Cheng, "Circularly polarized few-cycle optical rogue waves: rotating reduced Maxwell-Bloch equations," Physical Review E, vol. 88, no. 6, Article ID 062925, 2013.

[55] C. Z. Li, J. S. He, and K. Porsezian, "Rogue waves of the Hirota and the Maxwell-bloch equations," Physical Review E, vol. 87, no. 5, Article ID 012913, 2013.

[56] G. Mu and Z. Qin, "Dynamic patterns of high-order rogue waves for Sasa-Satsuma equation," Nonlinear Analysis: Real World Applications, vol. 31, pp. 179-209, 2016.

[57] S. H. Chen, "Twisted rogue-wave pairs in the Sasa-Satsuma equation,” Physical Review E, vol. 88, no. 2, Article ID 023202, 2013.

[58] B. L. Guo, M. Ling, and Q. P. Liu, "Nonlinear Schrödinger equation: generalized Darboux transformation and rogue wave solutions," Physical Review E, vol. 88, no. 2, Article ID 026607, 2012.

[59] X. Wang, J. Cao, and Y. Chen, "Higher-order rogue wave solutions of the three-wave resonant interaction equation via the generalized Darboux transformation," Physica Scripta, vol. 90, no. 10, p. 105201, 2015.

[60] X. Wang, Y. Li, and Y. Chen, "Generalized Darboux transformation and localized waves in coupled Hirota equations," Wave Motion, vol. 51, no. 7, pp. 1149-1160, 2014.

[61] D. F. Lawden, Elliptic Functions and Applicantions, SpringerVerlag, New York, NY, USA, 1989.

[62] K. W. Chow, "A class of doubly periodic waves for nonlinear evolution equations," Wave Motion, vol. 35, no. 1, pp. 71-90, 2002.

[63] J. S. Guo and B. Hu, "Quenching rate for a nonlocal problem arising in the micro-electro mechanical system," Journal of Differential Equations, vol. 264, no. 5, pp. 3285-3311, 2018. 